\title{
LA FLEXIBILIDAD EN LA PEQUEÑA EMPRESA: UN ESTUDIO RESPECTO AL DESARROLLO DE UN CONCEPTO OPERATIVO $Y$ CUANTITATIVO
}

Vicente Safón-Cano ${ }^{1}$

Juarez Perfeito ${ }^{2}$

Resumen: Ese artículo hace un abordaje de una temática muy desarrollada en el mundo académico que es la flexibilidad de las empresas delante de las variables del entorno. Específicamente es presentada una forma de cuantificación de esa flexibilidad en una pequeña empresa. Considerando los aspectos de los recursos de la empresa, comerciales, producción, financieros y de factor humano fue desarrollado un concepto operativo y cuantitativo de la flexibilidad empresarial.

Palablas-Clave: pequeña empresa, flexibilidad, variables del entorno.

\section{LA FLEXIBILIDAD EMPRESARIAL: ESTADO DE LA CUESTIÓN}

\subsection{LA VISIÓN TRADICIONAL}

La visión tradicional de la flexibilidad ha sido la más explotada en la literatura sobre management y la que con más asiduidad se encuentra en los textos actuales. Esta visión entronca muy bien con la concepción popular que se tiene del concepto, en la cual, atendiendo a la letra del Diccionario de la Real Academia de la Lengua Española, la flexibilidad significa: "calidad de flexible; disposición que tienen algunas cosas para doblarse fácilmente sin romperse; y disposición del ánimo a ceder y acomodarse fácilmente a un dictamen". Este sentido popular e intuitivo de flexibilidad se ha ido utilizando desde los primeros textos sobre la flexibilidad de la empresa, siendo difícil no encontrar su influencia en la literatura más moderna.

La contemplación demasiado simple de la realidad de la flexibilidad ha tenido dos efectos contrarios en la investigación: ha dificultado la construcción de una teoría consistente y global sobre la flexibilidad de las empresas; aunque, ha permitido un estudio pormenorizado y cuantitativo de áreas parciales de la empresa.

\footnotetext{
1 Universidad Valencia- España. Departamento de Administração.

${ }^{2}$ Universidade Regional de Blumenau, Programa de Pós Graduação em Administração, Blumenau/SC. E.Mail: perfeito@usj.edu.br
} 
La perspectiva clásica de la flexibilidad, o si se quiere, el espíritu más extendido del concepto de flexibilidad en los textos académicos, ha remitido y sigue remitiendo de forma invariable a una condición que se entiende deseable para cualquier empresa, máxime si ésta opera en entornos turbulentos.

Esta asunción de la bondad de la flexibilidad se ha explicado a partir del concepto de incertidumbre. Según escribió Thompson (1967), la exigencia de flexibilidad nace de la incertidumbre a la que se ven sometidos los sistemas abiertos, puesto que, en condiciones de certidumbre, donde se conoce con exactitud y certeza lo que ocurrirá, la flexibilidad es más bien indeseable, por cuanto se paga por algo que no se tendrá oportunidad de utilizar. Parece entonces lógico que conforme se pasa de una situación de certidumbre a una de riesgo, después a otra de incertidumbre, y de esta última a otra de ignorancia parcial (ANSOFF, 1976, p. 44-45, 70-ss), la empresa vaya asumiendo la necesidad de introducir dosis crecientes de flexibilidad en su management y recursos (figura 1); y no cabe la menor duda que esto se ve como una virtud deseable y positiva en la empresa. Frases como las de Hirst (1990, p. 106) permiten ver la concepción clásica de la flexibilidad:

"La flexibilidad puede considerarse como un seguro: un pago por el premio que supone mitigar el alto coste que podría sobrevenir si ocurriera algo indeseado".

\begin{tabular}{|l|l|l|l|}
\hline Condición & Alternativas & Probabilidades & Flexibilidad \\
\hline Certidumbre & Conocidas & Sucesos seguros & $\begin{array}{l}\text { Innecesaria y } \\
\text { negativa }\end{array}$ \\
\hline Riesgo & Conocidas & Conocidas & Positiva \\
\hline $\begin{array}{l}\text { Incertidumbre } \\
\begin{array}{l}\text { Ignorancia } \\
\text { parcial }\end{array}\end{array}$ & No conocidas & No conocidas & Necesaria \\
\hline
\end{tabular}

Figura 1- Condiciones de la toma de decisiones y necesidad de flexibilidad Fonte: Safón, 2000, p. 25

Una segunda cuestión relacionada con la anterior puede verse en el uso marginal dado a la flexibilidad por la literatura. Dentro de la perspectiva clásica, la flexibilidad se ha visto como un atributo de calidad en el proceso de toma de decisiones, donde la dirección de la empresa la incorporaba como objetivo a perseguir en la formulación de las políticas de la empresa (ANSOFF, 1976, p. 79-82). La comunión de la flexibilidad con la prudencia ha sido especialmente intensa conforme las empresas han ido enfrentándose a entornos y situaciones internas más complejas y turbulentas, llegándose a decir que los directivos deberían, ahora más que nunca, ser muy cautos con el posible fracaso, minimizando las consecuencias no esperadas o desastrosas, y sometiendo los objetivos de eficiencia a los de flexibilidad.

El mismo Ansoff retiene la interpretación clásica mencionada y concluye que la flexibilidad no debe entenderse como un objetivo en sí mismo e independiente de los objetivos fundamentales del management (beneficio, crecimiento, etc.), sino que debe ser justamente considerada como un objetivo de calidad en la toma de decisiones empresariales. En opinión del autor, la flexibilidad debe ser entendida como un tipo de respuesta para afrontar la incertidumbre que predomina en la relación empresa-entorno, ya que se trata de una respuesta preventiva que busca reducir los tiempos y los costes 
de la adaptación de la gestión a los nuevos acontecimientos sociales, políticos y tecnológicos (ANSOFF, 1976, p. 79-82).

Se observa, según la lógica expuesta, una visión de la flexibilidad como requisito en la toma de decisiones, de forma que, por ejemplo, debería elegirse una maquinaria con mayor capacidad de variar la producción (en volumen y tipos), aunque fuera, en alguna medida, menos eficiente, antes que otra con más rendimiento, pero con menos capacidad de variación; o que convendría estar presente en el desarrollo de diferentes tecnologías alternativas antes que en sólo una de ellas.

\subsection{PERSPECTIVAS DE LA FLEXIBILIDAD}

Las concepciones anteriores y la definición de flexibilidad clásica o popular están presentes en la mayoría de los textos sobre el tema, aunque también es cierto que cada vez más se leen ensayos con una perspectiva diferente y marcada por el acento dado a la importancia del aprendizaje en la organización, las nuevas oportunidades tecnológicas y la gran fuente de flexibilidad conseguida merced a la cooperación empresarial.

Tratando de hacer un estudio retrospectivo del asunto, es necesario advertir que la flexibilidad de la empresa ha sido un tópico que ha preocupado desde siempre a los managers de las organizaciones y a los académicos del management, por lo que no debe verse como una cuestión novedosa ni siquiera actual ${ }^{3}$. Como concepto y característica amplia de la realidad, la flexibilidad ha sido utilizada desde diversos puntos de vista y para explicar fenómenos diferentes, aunque, salvo raras excepciones, su perfil siempre ha sido el bautizado aquí como clásico.

Del examen de la literatura científica sobre el tema en cuestión se observa que se viene hablando de flexibilidad básicamente desde dos grandes posiciones. Una primera posición que hace referencia a la flexibilidad de la empresa y sus subsistemas, y a la flexibilidad conseguida merced a redes de empresas. Y una segunda posición que tiene que ver con sistemas económicos más complejos y más agregados que las simples organizaciones. Concretamente, la segunda corriente ha estado representada por los macroenfoques (a nivel de naciones) y los mesoenfoques (a nivel de regiones fundamentalmente) $)^{4}$.

Como es natural, estos cuerpos de literatura divergen debido a sus diferentes objetos formales y material de estudio. Sin embargo, ambas corrientes comulgan con una concepción parecida de flexibilidad, Ilamada aquí clásica. Empezando por la última, esto es, el macro y mesoenfoque, y sin ánimo de practicar un estudio exhaustivo, conviene advertir que los trabajos han girado en torno al fenómeno de la crisis mundial del fordismo y las políticas macroeconómicas keynesianas, buscando en sus investigaciones el diagnóstico y las posibilidades de desarrollo económico de regiones y naciones, basándose este desarrollo en modelos de flexibilidad organizativa, industrial y tecnológica. Exponentes de este planteamiento han sido muchos y variados estudios. Algunas obras que han gozado de gran difusión y lectura han sido las de Piore y Sabel (1984), Dore (1986),

\footnotetext{
${ }^{3} \mathrm{Ansoff}$ (1976, p. 81) recuerda que "la preocupación por alguna forma de flexibilidad externa es un fenómeno relativamente reciente [...] En contraste, la preocupación por la flexibilidad interna es tan vieja como el propio negocio".

${ }^{4}$ Los mesoenfoques son planteamientos que se encuentran entre las teorías macroeconómicas estructuralistas y los enfoques individualistas centrados en las empresas. Los mesoenfoques, llevados a cabo a través de mesoanálisis, tratan de estudiar los sistemas económicos en un nivel intermedio entre micro y macro (Gilly, 1994), como es el caso de las hileras de producción o los distritos industriales.
} 
Boyer (1986), Becattini $(1987,1989$,1990), Hirst y Zeitlin (1989, 1991), y Benko y Lipietz (1994). En todas ellas, como certeramente señalan Burrows, Gilbert y Pollert (1992, p. 12), el debate ha cristalizado, de un modo u otro, alrededor de tres conceptos: fordismo, postfordismo y flexibilidad.

En el enfoque de la flexibilidad que se centra en la empresa, la literatura ha asumido como objeto material de estudio a las empresas tomadas individualmente y evitando tratarlas como "cajas negras" . Este cuerpo de literatura también presenta grandes diferencias de enfoque (objetivos, métodos, disciplinas de soporte, etc.), pudiéndose identificar dos tipos de literatura en el estudio de la flexibilidad de la empresa: uno cuyo objetivo es el estudio positivo y normativo de la flexibilidad global de la empresa, tratando cuestiones relativas a la flexibilidad estratégica y estructural de manera integral, y tomando a la empresa como unidad de análisis o como parte de agregados más complejos (redes); y otro cuyo objetivo se centra en la investigación de los subsistemas particulares de la empresa, dedicando atención especial al subsistema de producción y técnico (flexibilidad productiva) y al subsistema social (flexibilidad del trabajo).

La figura 2 recoge esquemáticamente las relaciones vistas hasta ahora.

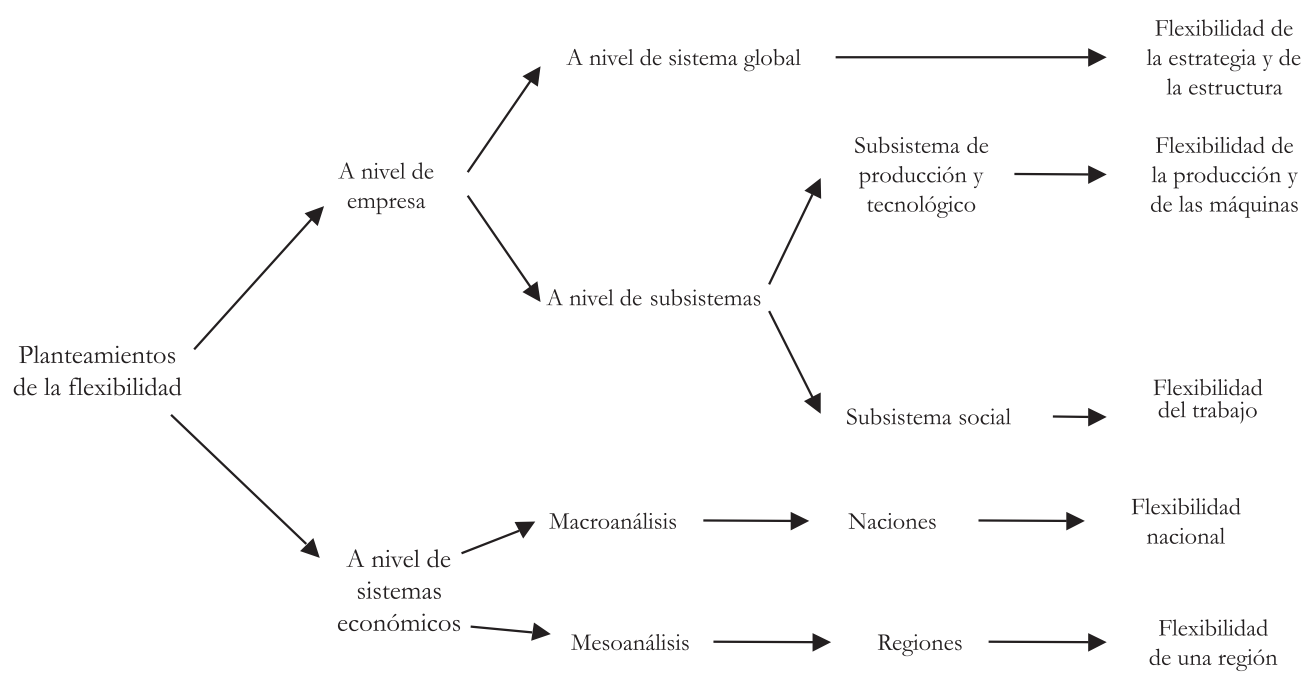

Figura 2 - Planteamientos dominantes en la literatura sobre flexibilidad Fonte: Safón, 2000, p. 28

La flexibilidad a nivel de empresa ha sido tratada por multitud de trabajos empíricos y teóricos. Sin embargo, hay que destacar que es tanta la pluralidad de ensayos como la diversidad y heterogeneidad de los conceptos trabajados y los resultados obtenidos. La primera y fundamental fuente de diversidad y confusión hay que buscarla en la propia definición de flexibilidad empleada en las investigaciones ${ }^{6}$. Este hecho ha sido destacado por parte de la literatura. Por ejemplo, Upton (1994) recoge en su estudio que las definiciones están a menudo coloreadas por situaciones empresariales o problemas particulares, y que tal heterogeneidad de acepciones no hace sino dificultar la construcción

\footnotetext{
${ }^{5}$ Esto último no es totalmente cierto, ya que gran parte de la literatura encuadrada en esta perspectiva, aunque ha asumido como objeto material de investigación a la empresa, no ha llegado a contemplar el fenómeno como de "caja traslúcida", y se ha quedado en un estudio de aspectos superficiales o de características sistémicas globales, sin entrar a tratar los mecanismos internos de la empresa que producen la flexibilidad.
} 
de un consenso epistemológico y una teoría general. También Evans (1991) arguye que el concepto de flexibilidad es polimórfico, en cuanto tiene un número diferente de significados en varios contextos, siendo, pues, un concepto contingente difícil de generalizar.

Tratando de superar la diversidad presente en las definiciones, el examen de la literatura sobre el tema de la flexibilidad permite ver que el concepto, a nivel general y global, y las dimensiones que lo definen, están presentes de manera generalizada en la gran mayoría de los casos examinados (aun cuando a veces se presenten de forma tácita). Esas nociones, ampliamente asumidas por la concepción clásica, permiten la siguiente definición provisional de flexibilidad propuesta por Safón (2000, p. 31):

a) la flexibilidad de la empresa se concibe, en sentido lato, como la capacidad de hacer frente al cambio. El cambio puede tener origen en el entorno y en el interior de la empresa, aunque la literatura privilegia el cambio de origen externo;

b) la flexibilidad se caracteriza por tres dimensiones,

- dimensión sistémica. Es decir, la capacidad que posee la empresa como sistema para hacer frente al principio de la variedad requerida, de modo que, en función de los requisitos del entorno, ofrezca prestaciones convenientes para su adaptación;

- dimensión económica. Que tiene que ver con el coste del cambio;

- dimensión temporal. Que remite a los tiempos de reacción.

En un ámbito más restringido, a nivel de subsistemas, la producción científica ha sido más prolífica que la anterior, quedando muy estudiados el sistema productivo y la

\footnotetext{
${ }^{6}$ Algunas muestras de las diferentes definiciones de flexibilidad se recogen a continuación:

- Hart (1937): La flexibilidad posibilita la modificación de un curso de acción en concordancia con una situación que de manera caprichosa nos obliga a desviarnos de las previsiones.

- Eppink (1978, p. 10): La flexibilidad de la empresa puede ser definida como "aquel atributo de la organización que la hace menos vulnerable o que la deja en una situación más apropiada para responder convenientemente a cambios imprevistos en el entorno".

- Mascarenhas (1981, p. 78): La flexibilidad es "la habilidad de la empresa para el cambio [...] La empresa puede ser inflexible cuando presenta una estructura rígida, unos costes fijos elevados y consecuentemente una incapacidad al cambio veloz; o ser flexible, cuando posee la habilidad para cambiar sus inputs, procesos y productos rápidamente".

- Aaker y Mascarenhas (1984, p. 74): La flexibilidad es "la habilidad de la empresa para adaptarse a los cambios del entorno que tienen un importante impacto en los resultados de la empresa".

- Harrigan (1985, p. 1): "La flexibilidad estratégica hace referencia a la habilidad de la empresa para 'reposicionarse' en un mercado, cambiar sus planes, o desmantelar sus actuales estrategias cuando el mercado servido ha dejado de ser atractivo".

- Bañegil (1993, p. 24): “Una organización flexible es aquélla que tiene capacidad de adaptación permanente y de reacción a tiempo a los cambios de su entorno competitivo".

- Upton (1994, p. 73): "La flexibilidad es la habilidad para cambiar o reaccionar con pequeñas perdidas de tiempo, esfuerzos, costes o performance".

- Koenes (1994, p. 38): "Flexibilidad es la capacidad que tienen las organizaciones para adaptar con rapidez sus valores, estrategias, habilidades, estructuras, sistemas, personal y estilo de gestión a los cambios que se producen en su entorno". - Sánchez (1995, p. 138): La flexibilidad de la empresa hace referencia a las "habilidades para responder a las varias demandas de los entornos competitivos dinámicos".

- Lund y Gjerding (1996, p. 13): "La flexibilidad es la capacidad, basada en estructuras y procesos de aprendizaje, para responder con nuevos productos y tecnologías al entorno cambiante".

- Albizu (1997, p. 11): "La flexibilidad empresarial es la capacidad general de adaptación de la empresa que le permite afrontar los cambios necesarios en orden a mantener/mejorar su posición competitiva".

- Volberda (1998, p. 100): "La flexibilidad es una condición que depende de la variedad de capacidades que posee una organización y de la velocidad con la que las puede activar".

La mayoría de las definiciones de los autores anteriores, entienden la flexibilidad como capacidad de cambio, sometida ésta a unos costes (del propio cambio) y unos tiempos de reacción (tiempo para descubrir la necesidad del cambio y que ha de transcurrir hasta que el cambio es efectivo).
} 
tecnología empleada ${ }^{7}$, el área comercial $^{8}$, el ámbito financiero ${ }^{9}$ y el factor humano ${ }^{10}$.

El estudio de la flexibilidad a nivel de subsistemas es el que pone de manifiesto con más claridad la concepción clásica de la flexibilidad. Este nivel de análisis ha permitido conocer las particularidades de la empresa en lo tocante a su ámbito interno, aunque ha perjudicado la construcción de una teoría general de la flexibilidad empresarial. Sin embargo, no por ser una perspectiva limitada deja de ser valiosa, puesto que los trabajos centrados en las áreas funcionales son el punto de partida para desarrollar una propuesta de cuantificación de la flexibilidad de la empresa. Vale la pena comentar, aunque sea únicamente una primera indicación, que una cosa es definir la flexibilidad y decir cómo se consigue, y otra pretender medirla o cuantificarla.

\subsection{HACIA LA SUPERACIÓN DE LA PERSPECTIVA CLÁSICA}

El problema fundamental que presenta la perspectiva clásica en la definición de la flexibilidad es que cuando se plantea el estudio de la empresa a nivel global, habla de la flexibilidad como una característica organizativa y estratégica demasiado general o para circunstancias muy particulares, no llegando a penetrar totalmente en el ámbito interno de la empresa. Y cuando el nivel de análisis se traslada a las partes (subsistemas), entonces se parcela demasiado, perdiendo la perspectiva de conjunto, olvidando las relaciones, y desvirtuando el concepto de flexibilidad en ciertos atributos técnicos $u$ operativos. Es por ello que la definición recogida más arriba, si bien no supone un paso en falso en la construcción de una teoría más general y aceptada, no es suficiente para orientar el management de las organizaciones actuales.

Por todo lo visto, puede concluirse que no parece que exista una teoría de la flexibilidad de la empresa global y aceptada con generalidad. El fenómeno permanece estudiado todavía en un estadio disperso y fraccionado, que impide (o dificulta) asumir cualquier supuesto para utilizarlo más allá del contexto en el cual ha sido formulado. En este estado de cosas, el presente artículo pretende extraer aquello que sea generalizable de cada aportación, e intentar vertebrarlo en un planteamiento global y coherente.

Desde la perspectiva de este trabajo, y siguiendo la propuesta de Safón (2000, p. 33), "el punto de partida para analizar la flexibilidad debe ser su concepción como capacidad de adecuación del sistema-empresa a la complejidad (variedad y variabilidad) del contexto interno y externo, de forma rápida y económica". En consecuencia, no está en nuestro ánimo alejarnos de los enfoques convencionales practicando una teoría ex novo, sino que nuestra intención es llevar la flexibilidad a un terreno más fructífero y explicativo del que ha sido presentado hasta ahora. Un terreno que tiene que ver con el gobierno de la complejidad y el postfordismo, y que trata de hacer converger la literatura más amplia acerca del postfordismo, con la más específica que habla de flexibilidades de subsistemas empresariales particulares (finanzas, producción, etc.).

\footnotetext{
${ }^{7}$ Ejemplos de estas aportaciones son los trabajos de Stigler (1939), Gerwin (1982, 1987, 1989 y 1993), Zelenovic (1982), Slack (1983 y 1987), Jaikumar (1984), Wheelwright y Hayes (1985), Ferré (1988), Cox (1989), Bañegil (1993), Upton (1994), y Suárez, Cusumano y Fine (1995).

${ }^{8}$ Algunos ejemplos son los estudios de Gerwin y Tarondeau (1984), Blois (1985), Jaikumar (1986), y Anderson y Narus (1995).

${ }^{9}$ Ejemplo de este interés son los trabajos de Donaldson (1969), Goldman (1978), Jones y Ostroy (1984), Mason (1986), Connell y Phillips (1988), y Kulatilaka (1993).

${ }^{10}$ Entre otros: Atkinson (1984 y 1987), Nacional Economic Development Office (1986), Atkinson y Meager (1986), Atkinson y Gregory (1986), OCDE (1991), Fina (1991), Sarfati y Kobrin (1992), Martín (1993), Skyrme (1994), Procter et al. (1994), y Albizu (1997).
} 
Una forma interesante de reconducir el problema de la definición de flexibilidad empresarial puede encontrarse en la Teoría General de Sistemas. Ésta, en su rama conocida como teoría de sistemas flexibles, considera un sistema como ${ }^{11}$ :

"Una porción del mundo que se percibe como una unidad, y que puede mantener su identidad, a pesar de los cambios de éste".

Y define los sistemas flexibles como aquellos sistemas (Gigch, 1990, p. 82):

Que pueden adoptar algunos estados debido a las condiciones del medio e incluso preservar sus identidades originales a pesar de estas influencias. El sistema solar, una fuente, una familia, una colmena, una ciudad, una nación, y una firma de negocios, son sistemas que pasan por cambios continuos en sus elementos componentes y probablemente en su forma percibida. Los sistemas definidos como flexibles poseen una estructura, reaccionan al medio mediante el cambio de sus funciones a corto plazo, pasan por cambios lentos a largo plazo, pero mantienen su identidad y evolucionan.

En el sistema, la rigidez y la flexibilidad llegan a ser dos caras de la misma moneda. Las reglas de la estabilización sistémica son las que permiten hacer posible variar el comportamiento corriente (adaptación). Paralelamente, ninguna regla podría mantenerse invariable si no fuera capaz de garantizar una cierta eficacia en la respuesta a las contingencia ambientales (evolución). Por esto, desde el punto de vista sistémico, la flexibilidad puede entenderse como el producto de normas organizativas tejidas para gobernar los comportamientos colectivos, caracterizados por un elevado nivel de interdependencia, de modo que se les permita exhibir una cierta variedad (diversidad y diferenciación) y/o variabilidad (transformación en el tiempo), manteniendo un grado aceptable de coherencia (GIGCH, 1990; LILIENFELD, 1991).

Esta breve referencia a la TGS, desarrollada más extensamente en otro trabajo previo (Safón, 2000), nos permite concluir que la definición general de flexibilidad se debe construir a partir de dos ideas centrales (Safón, 2000, p. 68): "lo ofrecido por la empresa", lo cual caracteriza la flexibilidad como la extensión de la oferta de variedad y variabilidad que resulta económicamente gobernable con base en un cierto sistema de reglas (invariantes); y la "base de invariantes y su evolución", y por consiguiente, los cambios en la oferta de variedad y variabilidad que pueden ser gobernados por la empresa.

\section{DESARROLLO DEL CONCEPTO OPERATIVO Y CUANTITATIVO DE LA FLEXIBILIDAD EMPRESARIAL}

\subsection{LA FLEXIBILIDAD DE LOS RECURSOS DE LA EMPRESA}

Los recursos de la empresa son la base para desarrollar las capacidades que construyan la ventaja competitiva de la estrategia de la empresa (Grant, 1995). Es por ello que su análisis es fundamental por ser los cimientos sobre los que descansa la estrategia de la empresa y por ende su flexibilidad.

Una forma sencilla de analizar la flexibilidad de los recursos es relacionarla con dos atributos que caracterizan a todo recurso:

\footnotetext{
${ }^{11}$ La cita se encuentra en Gigch (1990, p. 82), el cual transcribe un trabajo de Rapoport de 1970.
} 
a) la posibilidad de que un recurso tenga varios usos en un momento dado (polivalencia);

b) la posibilidad de que un recurso sea sustituido por otro, ya sea porque el primero no ofrece el resultado perseguido o simplemente porque se desea prescindir de él (sustituibilidad) $^{12}$.

De esta manera se tienen dos formas de comportamiento de los recursos de una empresa y dos tipos de medidas para la cuantificación de su flexibilidad: el grado de polivalencia (número de usos posibles) y el grado de sustitución (posibilidad de eliminar un recurso y sustituirlo por otro $)^{13}$. Definidas estas dos vertientes de la flexibilidad de los recursos, puede deducirse que una empresa posee unos recursos más flexibles en la medida que éstos pueden adaptarse más fácilmente a diferentes usos posibles, y cuanto más rápido y económico es su proceso de sustitución.

El hecho que los recursos planteen simultáneamente dos fenómenos distintos a nivel de flexibilidad complica el análisis de su flexibilidad global. En efecto, la doble consideración de la flexibilidad en los recursos obliga a contemplar situaciones contradictorias. Por ejemplo, un caso que puede presentarse es el de un recurso muy flexible en el uso, pero que sin embargo es muy difícil sustituirlo, y por tanto es inflexible en cierta medida; o el caso inverso: un recurso poco polivalente pero fácilmente sustituible. Esta realidad dual en el comportamiento de la flexibilidad de los recursos, que más bien es la regla que la excepción, dificulta cualquier iniciativa en la medición global de su flexibilidad, y obliga a ser muy cautos en los análisis que tratan el fenómeno de la flexibilidad empresarial sin hacer mención a esta particularidad (figura 3).

\begin{tabular}{|l|l|l|l|}
\hline & & \multicolumn{2}{|c|}{ Polivalencia } \\
\hline & & Pequeña & Grande \\
\hline \multirow{3}{*}{ Sustitución } & Fácil & $?$ & $\begin{array}{l}\text { Recurso } \\
\text { flexible }\end{array}$ \\
\cline { 2 - 4 } & Difícil & $\begin{array}{l}\text { Recurso } \\
\text { inflexible }\end{array}$ & $?$ \\
\hline
\end{tabular}

Figura 3 - Clasificación de los recursos según su polivalencia y grado de sustitución Fonte: Safón, 2000, p. 167

\footnotetext{
${ }^{12}$ El concepto de sustituibilidad de los recursos adoptado aquí es más amplio que el de liquidez de los recursos, entendida por Ansoff (1976) como medida de la flexibilidad interna de una empresa. En efecto, Ansoff (1976, p. 79-ss) sostiene que la flexibilidad externa, en contraposición a la interna, puede ser conseguida a través de un modelo diversificado de las inversiones efectuadas en los productos y los mercados (como él mismo recoge, "no poner todos los huevos en un mismo cesto"); y la flexibilidad interna mediante la liquidez de los recursos empresariales. En nuestra opinión, parece más adecuado relacionar el concepto de sustituibilidad no sólo con la existencia de recursos líquidos, sino también con las oportunidades de desmovilización de inversiones, de rescisión de vínculos operativos relacionados con situaciones contractuales y con la resolución de acuerdos con otras organizaciones externas. Por otra parte, el verdadero obstáculo al cambio no es el financiero, o sea, la disponibilidad de fondos o la capacidad de endeudarse, sino el económico, es decir la oportunidad de adaptarse y sustituir los recursos de forma económica y rápida.

${ }^{13}$ Esta propuesta de flexibilidad es algo más amplia que la noción de flexibilidad de los recursos de Sánchez (1995), por cuanto considera no sólo los usos alternativos, los costes y dificultades del cambio, y el tiempo necesitado para el uso alternativo del recurso (Sánchez, 1995, p. 139), sino también la posibilidad de sustitución de los recursos. Nuestra perspectiva, que une en la flexibilidad de los recursos la polivalencia y la capacidad de sustitución, coincide bastante con la teoría de los recursos y capacidades. A este respecto, Ventura (1996, p. 105-106) indica que "la perspectiva de la teoría de los recursos y capacidades requiere diferencias entre recursos versátiles o no específicos al tipo de estrategia y entorno en que se actúa, y aquellos recursos que son más dependientes o específicos de la orientación estratégica actual, y que por tanto presentan costes hundidos si la estrategia de la organización se modifica de manera clara [...] Los recursos del primer tipo aumentan los grados de libertad para adaptarse a los cambios, mientras que los segundos limitan la capacidad de elección y aumentan la dependencia respecto a la trayectoria seguida previamente".
} 
Otro problema al analizar de esta manera a los recursos de la empresa es que su naturaleza no es homogénea. Como recoge Grant (1995), los recursos se clasifican en tres categorías:

a) recursos tangibles (físicos y financieros);

b) recursos intangibles (tecnología, reputación y cultura);

c) recursos humanos (conocimientos y destrezas especializadas, capacidades de comunicación y relación, y motivación).

Los recursos físicos son un tipo de recurso que permite muy bien el análisis de la polivalencia y sustitución. Por ejemplo, la fábrica, la infraestructura de venta, los almacenes, etc., tienden hoy a ser dotados de un mayor grado de flexibilidad porque, sobre todo desde hace algún tiempo, ya vienen concebidos en su estructura y modalidad de funcionamiento para responder a una variedad de actividad más amplia y diferenciada. Concretamente, la flexibilidad de la fábrica, obtenida mediante la llamada automatización flexible, y sobre la base del principio de la integración flexible, representa una de las bazas más importantes en el programa de flexibilización del sistema productivo de las empresas actuales. Por otro lado, los recursos físicos clasificables como de circulantes, o sea, las materias primas, los materiales de consumo, piezas de recambio, etc., pueden representar un vínculo, y por consiguiente, un factor de inflexibilidad (ya que sus usos son limitados y cabe cierta dificultad en su liquidación). Sin embargo, debido a su poca importancia relativa (respecto al total de ventas o de activos contables), los recursos circulantes no deberían provocar problemas de falta de flexibilidad. Paralelamente, los recursos financieros no tienen por qué representar un elemento de vínculo, ya que, en la mayoría de los casos, están a la entera disposición de la gestión, siendo uno de los activos más flexibles por su polivalencia y movilidad.

El problema aparece cuando se trata de aplicar el análisis de la capacidad polivalente y de sustitución a los recursos que forman parte de los invariantes de la empresa (intangibles y sobre todo los humanos). En estos casos el argumento anterior de que un recurso es flexible cuando es polivalente y fácilmente sustituible pierde fuerza y aplicación. Además, si se considera que la relación que existe entre la estrategia y los recursos está mediada por las competencias ${ }^{14}$, es todavía más difícil asegurar que un recurso supuestamente inflexible influirá negativamente en la flexibilidad global de la empresa.

Los recursos intangibles y los humanos son la base fundamental sobre la que se desarrollan las competencias de las empresas. Estos recursos, sobre todo los humanos y el carácter institucional de la organización, son el principal elemento invariante de la empresa que garantiza la reproducción de competencias que, a su vez, alimentan la ventaja competitiva y permiten la adaptación de la empresa. Por ello no conviene generalizar el análisis de la flexibilidad de los recursos a todos los recursos en posesión de la empresa, ya que algunos ofrecerán la necesaria flexibilidad proporcionando una elevada polivalencia y capacidad de sustitución (por ejemplo una máquina universal), y otros harán lo propio siendo manifiestamente inflexibles (por ejemplo, la capacidad comunicativa a nivel interno y externo de la organización).

${ }^{14}$ Este razonamiento está muy desarrollado en la obra de Grant (1995). 


\subsection{LA FLEXIBILIDAD COMERCIAL}

La literatura sobre marketing acostumbra a separar las decisiones comerciales de la empresa en cuatro grandes capítulos: decisiones sobre precios, productos, distribución y comunicación. Siguiendo ese esquema, la flexibilidad comercial puede medirse en función de que ese amplio conjunto de decisiones pueda ser adaptado, dando diferentes usos a los recursos ya existentes, y/o sustituido por decisiones basadas en recursos totalmente nuevos.

Comenzando por los precios, una empresa presenta flexibilidad en los precios si es capaz de modificarlos rápida y económicamente. En efecto, una empresa disfruta de flexibilidad en los precios si:

a) se le permite el cambio de precios por no tener restricciones legales a la variación ni bandas de fluctuación, y además no está sometida al poder de los clientes (ni de ninguna otra fuerza) que le imponga los precios en las transacciones comerciales;

b) los cambios de precios son trasmitidos velozmente, de modo que la demanda pueda ajustarse lo más rápidamente posible a los nuevos niveles de precios;

c) el cambio de precios no supone un coste excesivo (en comunicación de la nueva oferta) por tener que proceder en tiempos relativamente cortos ${ }^{15}$.

Respecto a los productos, una empresa presenta flexibilidad en los productos si:

a) posee una gama de productos amplia ${ }^{16}$;

b) es capaz de modificarlos y/o sustituirlos rápida y económicamente. Esta flexibilidad está condicionada por tres elementos esenciales,

- la existencia de una gama de productos amplia y la capacidad de la empresa de gestionarla.

- la capacidad de la empresa para descubrir y desarrollar nuevos productos e intuir los cambios de las preferencias de los consumidores, que pueden suponer la eliminación de productos, la creación de nuevos productos o la modificación de los existentes ${ }^{17}$;

- las condiciones de flexibilidad del sistema productivo, sobre todo de la flexibilidad de producto, puesto que aunque se cumpla la condición anterior respecto a la capacidad de descubrir y desarrollar nuevos productos o cambios en los existentes, la modificación o sustitución de productos depende de las posibilidades reales y potenciales del sistema productivo de la empresa, a nivel de tipologías de productos y velocidad en la introducción de nuevos productos ${ }^{18}$.

\footnotetext{
${ }^{15}$ En relación con los puntos dos y tres, conviene subrayar que la velocidad y economía del cambio de precios depende de la eficacia de la estrategia de comunicación de la empresa (publicidad, fuerza de ventas, relaciones públicas, etc.) y del canal de distribución utilizado para comercializar los productos.

${ }^{16}$ La gama de la empresa puede estar formada por productos fabricados por ella, pero también por productos comprados a otras empresas y vendidos sin transformación.

${ }^{17}$ En este nivel de análisis la flexibilidad de los productos está muy condicionada por el resto de la empresa, ya que la capacidad requerida para descubrir y desarrollar nuevos productos o cambios en los existentes suele requerir el trabajo coordinado de varios departamentos organizativos (ingeniería, comercial, producción e I+D).

${ }^{18}$ La sumisión de la flexibilidad comercial al sistema de producción es un tema que ha interesado tradicionalmente a los expertos en management. Un ejemplo de las relaciones de dependencia con las que se encuentra el sistema comercial, a nivel de producto, puede examinarse en el clásico trabajo de Shapiro (1977).
} 
En lo tocante a la flexibilidad de la distribución, una empresa es tanto más flexible cuanto más fácilmente puede:

a) cambiar los términos de la relación contractual: períodos de entrega, plazos de pago, servicio al cliente, introducción de nuevos productos, y en general, todo lo que gira alrededor del producto y la relación empresa-distribuidor;

b) sustituir el canal de distribución por otro distinto; todo ello en el menor tiempo posible y con unos costes razonables.

Las empresas actuales están flexibilizando sus redes de distribución de formas muy diversas. No obstante, en la normal variedad de estrategias evidenciadas en la práctica, puede observarse una cierta tendencia hacia la externalización de la función de distribución. Esta estrategia se basa en acuerdos menos vinculantes que la inversión directa, pero con mayores obligaciones que la simple distribución realizada por terceros mediante una relación transaccional. Respecto a este comentario, el fenómeno anterior puede ilustrarse con el crecimiento exponencial de las franquicias, que suponen una opción intermedia entre la total internalización y la total externalización de la función de distribución.

Finalmente, la comunicación de la empresa se manifiesta flexible cuando permite la modificación y sustitución de sus elementos, sin causar graves perjuicios a la empresa por costes o demoras excesivos. El estudio de la flexibilidad de la comunicación obliga a comprobar el grado de vinculación y versatilidad de la fuerza de ventas, y las posibilidades de modificación o cambio del tipo de publicidad, la imagen transmitida, la promoción de ventas y las relaciones públicas. Así, por ejemplo, una buena flexibilidad en la comunicación debe poder conseguir un cambio rápido y económico en la imagen de cierto producto, añadir nuevas ofertas a las ya existentes en la fuerza de ventas, o modificar las relaciones que se mantienen con los medios publicitarios.

Asimismo, para ser completo, el análisis de la flexibilidad comercial debe recoger el estudio de los clientes y proveedores, aunque estos últimos suelen tratarse en la flexibilidad productiva. Respecto a esto, una empresa es más flexible cuanto mayor número de clientes independientes (de diferente naturaleza y a ser posible sin relación entre ellos) acaparan una importante cifra de la facturación de la empresa; y cuanto mayor es el número de alternativas en las fuentes de aprovisionamiento (diversos proveedores intercambiables entre sí).

\subsection{LA FLEXIBILIDAD PRODUCTIVA}

La flexibilidad productiva o del subsistema de producción ha sido muy estudiada y desarrollada en la literatura sobre management. A pesar de la gran heterogeneidad y confusión presente en los estudios sobre el tema, se observa una cierta coincidencia al definir la flexibilidad productiva en términos de nivel y características de la producción. El esquema que aquí va a utilizarse recoge el espíritu de casi todos los textos relativos a la flexibilidad productiva, e incluye las dimensiones económica y temporal necesarias para ofrecer buenas medidas de la flexibilidad. El trabajo que fundamentalmente se ha utilizado ha sido el de Slack (1983), el cual propone un modelo de medición de la flexibilidad productiva amplio y sencillo basado en tres parámetros:

a) la amplitud de los estados posibles, es decir, la capacidad de variación del output productivo a nivel de tipología de los productos, calidad de los productos y volumen de producción eficiente; 
b) el coste de la adaptación (economía de las variaciones);

c) el tiempo de la adaptación (velocidad de las variaciones).

De esto se desprende que un sistema productivo es tanto más flexible cuanto:

a) mayores son las variaciones posibles del output, en número y calidad ${ }^{19}$;

b) menores son los costes de los cambios;

c) más rápidos son los cambios.

Cualquier empresa de cualquier época ha conjugado de forma distinta los tres parámetros que definen la flexibilidad productiva (variedad de la producción, y economía y velocidad de las variaciones). Actualmente las empresas tratan de flexibilizar sus operaciones y tecnologías productivas con el uso de diferentes estrategias. Dentro de la abundante casuística, algunas estrategias empleadas con frecuencia son (Ferré, 1988; Carlsson, 1989; y Bañegil, 1993): la adopción de estructuras industriales polivalentes, adaptables a tecnologías alternativas; la búsqueda y aplicación de nuevos modelos de organización del trabajo en la fábrica; la negociación con proveedores y clientes de los plazos de entrega, y la creación de relaciones de cooperación; la disponibilidad de diferentes proveedores para los mismos materiales; el trabajo flexible y extraordinario; la subcontratación de capacidad y especialidad; el diseño modular de las plantas de producción, que se amplían sucesivamente, acomodándose al incremento de la demanda; y la reserva de capacidad o disponibilidad de factores productivos de reserva.

En relación con la estrategia recogida en último lugar, la reserva de capacidad o disponibilidad de factores de reserva suele citarse como la forma más tradicional de hacer frente a las oscilaciones de la demanda. En esta estrategia es muy habitual la fabricación para el inventario, mediante la cual se adapta la producción más o menos constante a los pedidos estacionales y se amortigua un plazo de fabricación excesivamente largo como para hacer frente a un pedido urgente, a productos defectuosos, o a averías de la maquinaria (Bañegil, 1993). No obstante, la fabricación para el inventario es una opción muy controvertida y criticada, puesto que al mismo tiempo flexibiliza e incrementa los costes de la empresa. Las variaciones positivas de la demanda (incrementos superiores a la capacidad productiva) pueden ser cubiertas inmediatamente con el recurso a los almacenes de productos acabados, permitiendo la adaptación de la empresa a los pedidos. En ese caso el stock de productos acabados es un elemento que contribuye a la flexibilidad de la empresa, siendo tanto mayor su contribución cuanto mayor sea su nive ${ }^{20}$. Sin embargo, la opción de recurrir a los almacenes de productos terminados implica la asunción de costes y riesgos importantes, a saber:

a) Coste de oportunidad de la inversión en almacenes;

b) Financiación, mantenimiento y deterioro de los almacenes;

c) Pérdida de ventas provocada por cambios en el mercado, como por ejemplo un cambio de gustos en los compradores o la aparición de productos nuevos que dejen obsoletos los actuales.

\footnotetext{
${ }^{19}$ Una empresa es más flexible si es capaz de producir más productos o categorías de productos distintos, y cuando las diferencias entre productos o categorías de productos son mayores.

${ }^{20}$ Por ejemplo, podría usarse como medida de flexibilidad el ratio stock mensual medio/volumen de producción mensual.
} 
Debido al doble efecto de la fabricación para el inventario, la empresa tiene que hallar una solución de compromiso en la determinación de su nivel de stocks. Por ello, para determinar el nivel de almacenes hay que tener presente que:

a) si se desplazan los stocks hacia adelante (menos materias primas y productos semiterminados, y más productos terminados), las consecuencias son,

-reducción del tiempo de respuesta;

-incremento del capital invertido;

-reducción de la capacidad para diferenciar los productos ya acabados;

b) si se desplazan los stocks hacia el origen del ciclo productivo (menos stocks de productos terminados, y más de semiterminados y primeras materias), las consecuencias son las contrarias, pero siempre, y en las dos opciones, implicando simultáneamente flexibilidad e inflexibilidad.

Sintetizando, la determinación del nivel de los almacenes, tanto de productos acabados como de productos intermedios y materias primas, pone en juego la flexibilidad del ámbito productivo y los resultados económicos de la empresa, obligándola a realizar una buena gestión de los almacenes, y en cualquier caso, obligándola a descartar soluciones simplistas que no integren la política de inventarios en otras más integrales (por ejemplo el just in time).

Otra vía de flexibilización del sistema productivo son los nuevos enfoques técnicos y organizativos. Sobre este particular, en el estudio de la flexibilidad productiva es obligatoria la visita al llamado lean management ${ }^{21}$. Este nuevo macroenfoque, en una óptica de integración de técnicas dispersas, y con un triple objetivo de incremento de la productividad, la calidad, y la flexibilidad productiva y comercial, se plantea como una filosofía de intervención a nivel productivo y de desarrollo de productos. Algunos ejemplos de sus principios se recogen seguidamente (CUSUMANO, 1994, p. 28):

a) principios para la producción (modelo Toyota);

- producción just in time;

- inventarios intermedios mínimos;

-concentración geográfica de ensambladores y productores de partes componentes;

-sistema de demanda pull con tarjetas Kankan;

-producción nivelada;

-velocidad en la organización/reconfiguración;

-racionalización de equipos y líneas;.

-trabajo estandarizado;

-dispositivos de automatización simples;

-trabajadores multiprofesionalizados;

${ }^{21} \mathrm{El}$ lean management es un estilo japonés de producción y desarrollo de productos. Esta denominación la llevó a cabo Krafcik (1988) en el marco de una caracterización dicotómica de los sistemas de producción entre buffered y lean, a su vez realizada sobre otra dualidad parecida propuesta por Shimada y MacDuffie entre robust y fragile (Krafcik, 1988: 44 y 51). 
-altos niveles de subcontratación;

-uso selectivo de la automatización;

- procesos de mejora continua;

b) principios para el desarrollo de productos (modelo Honda),

-rápida sustitución de modelos;

-frecuente expansión de la línea de productos;

-superposición y compresión de las fases de desarrollo;

-elevados niveles de suministro de trabajos de ingeniería;

-directores de proyecto muy influyentes;

-diseño de equipos y continuidad de los managers;

- estrictos programas de ingeniería y disciplina de trabajo;

-buenos y hábiles mecanismos de comunicación;

-equipos multiprofesionalizados de diseño e ingeniería;.

-diestro uso de herramientas CAD;

-mejoras continuas e incrementales en el producto.

Los nuevos enfoques técnicos y organizativos, entre los cuales está el Lean Management, han puesto de manifiesto que la flexibilización del sistema productivo está condicionada por la adaptación del factor humano a las nuevas exigencias tecnológicas y organizativas. Las crecientes posibilidades que abren los nuevos desarrollos tecnológicos ${ }^{22}$ implican la necesaria evolución del factor humano encargado de producir en el nuevo escenario tecnológico y productivo. En particular, el diseño de puestos y fundamentalmente la formación de los operadores que deben interactuar con las nuevas tecnologías pasa por (Jaikumar ${ }^{23}$, 1986; Adler, 1988; y Lehner, 1991):

a) una extensión de las tareas, tanto en sentido horizontal como en sentido vertical (enriquecimiento del trabajo).

b) un entrenamiento para el trabajo en grupo.

c) una mayor familiaridad con la tecnología de la información, especialmente con los soportes y lenguajes informáticos.

No sirve de mucho incrementar la sofisticación del sistema productivo (automatización, robotización e informatización) sin desarrollar el diseño de la organización, y dotar de autonomía y capacidad a los trabajadores que manipulan la tecnología pretendidamente "flexible". Dice Krafcik (1988) que, adicionalmente a la introducción de tecnologías flexibles, debe invertirse en el desarrollo de un nuevo management de la producción (lean production system), como condición necesaria para

\footnotetext{
${ }^{22}$ Diseño asistido por ordenador (CAD), producción asistida por ordenador (CAM), producción integrada por ordenador (CIM), sistemas de producción flexible (FMS) e intercambio electrónico de datos (EDI), entre otros.

${ }^{23}$ Merece la pena subrayar la gran importancia que concede este autor al papel del conocimiento en la defensa y creación de ventajas competitivas. Un conocimiento que no en vano se consolida con el aprendizaje experimental.
} 
avanzar paralelamente por los caminos de la flexibilidad y la alta performance ${ }^{24}$. Es decir, no sólo se deben adaptar e incorporar tecnologías flexibles de producción, sino también tecnologías de gestión, para que el resultado sea una mayor capacidad de respuesta y resolución de problemas internos, al tiempo que se consiguen buenos resultados económicos. Como recuerda Grant (1996, p. 192):

La flexibilidad no es principalmente un problema de las capacidades técnicas asociadas a la planta, instalaciones y otro tipo de equipamiento; es básicamente un resultado del 'software organizativo' -la estructura de la organización, los sistemas de toma de decisiones, la amplitud del diseño de tareas y las actitudes.

También coinciden Suárez, Cusumano y Fine (1995) con este último argumento. Apoyados en una investigación empírica, han puesto de manifiesto que la flexibilidad productiva (ellos la miden en función de las variables mix producido, velocidad en la generación de nuevos productos y volumen producido) depende mucho más de factores no tecnológicos que tecnológicos. En opinión de estos autores, factores como los grupos de trabajo temporales, las relaciones con proveedores y subcontratistas, y la extensión de la utilización de los componentes en el proceso de diseño de productos, hacen más por la flexibilidad que los puramente tecnológicos.

\subsection{LA FLEXIBILIDAD FINANCIERA}

La flexibilidad financiera se define como la capacidad de adaptación de los recursos financieros de la empresa a las exigencias del management, que se derivan esencialmente de acontecimientos imprevistos ${ }^{25}$. La flexibilidad financiera, de acuerdo con la definición anterior, está ligada a dos características:

a) el grado de liquidez y movilidad;

b) el grado de elasticidad.

En primer lugar, el grado de liquidez y movilidad define la medida según la cual una necesidad imprevista puede ser afrontada con los recursos propiedad de la empresa. Para su cálculo es necesario separar la liquidez de la movilidad. Por una parte, la liquidez se mide a través del fondo de maniobra o capital circulante. Y por otra parte, la movilidad se obtiene al observar el activo fijo que puede realizarse (convertirse en dinero) para hacer frente a las nuevas necesidades, con la restricción de no comprometer la actividad actual. En segundo lugar, el grado de elasticidad financiera o de expansión de los recursos financieros remite a la posibilidad de hacer frente a una necesidad no prevista recurriendo al mercado financiero. La elasticidad se define por la oportunidad cualitativa y cuantitativa de expansión de la estructura financiera (pasivo de la empresa). La figura 4 recoge las dos características de la flexibilidad financiera enunciadas anteriormente.

\footnotetext{
${ }^{24} \mathrm{El}$ objetivo actual del área de producción es conciliar la eficiencia económica, exigida por la fuerte competencia, con la flexibilidad necesaria para hacer frente a la turbulencia del entorno (Gerwin, 1993; Hayes y Pisano, 1994; y Sánchez, 1995). Hasta el nacimiento de las tecnologías flexibles (CAD/CAM, CIM y FMS) el sistema productivo se hallaba permanentemente inmerso en la disyuntiva entre eficiencia (costes de producción) y flexibilidad (variedad de los outputs productivos); problemática bien conceptuada en los trabajos de Woodward (1965), Abernathy (1978), y Hayes y Wheelwright (1979), los cuales se hacían eco de este peculiar trade-off. Sin embargo, con la emergencia e integración de las tecnologías flexibles y de la información, y las nuevas tecnologías organizativas basadas en el grupo, la formación y la autonomía, muchos investigadores se han cuestionado el anterior "intercambio" (Adler, 1988; Kotha y Orne, 1989; y Suárez, Cusumano y Fine, 1995), y han concluido que el dilema entre flexibilidad y eficiencia, aunque no completamente resuelto, ha superado la situación anterior, de modo que puede avanzarse, gracias a las tecnologías flexibles y organizativas, al mismo tiempo en ambas direcciones: hacia la variedad de outputs (tipologías, calidades y volúmenes) y la reducción de costes unitarios. ${ }^{25}$ La definición propuesta está inspirada en el concepto de movilidad financiera de Donaldson (1969) y flexibilidad interna de Ansoff (1976).
} 


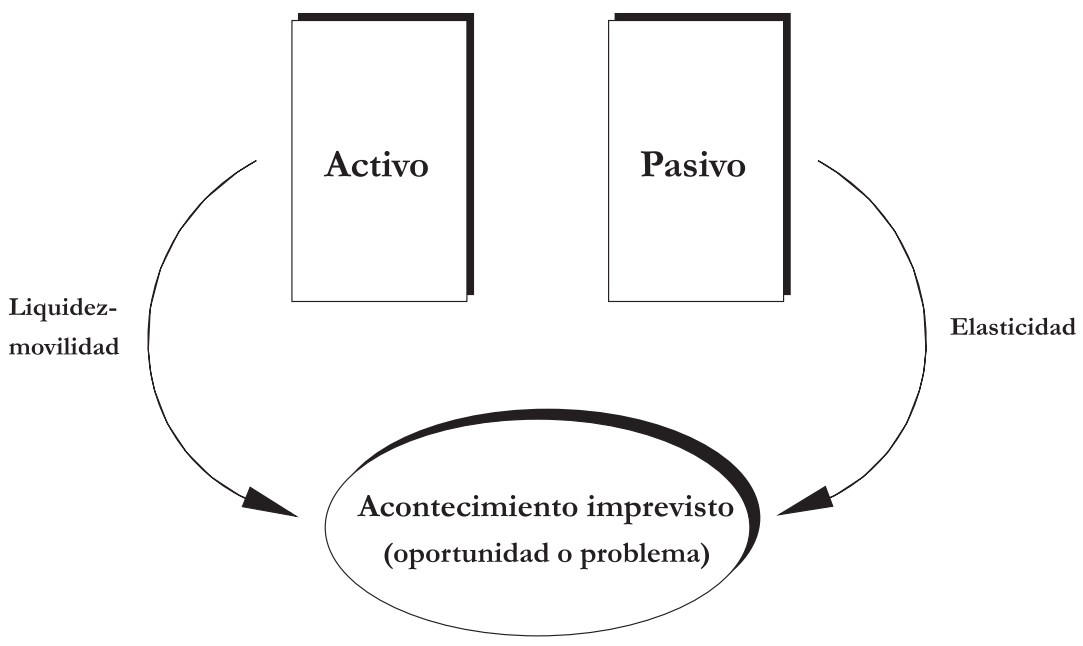

Figura 4 - Noción de flexibilidad financiera

Fonte: Safón, 2000, p. 180

Con base en la definición de flexibilidad financiera y la descripción de sus dimensiones, puede concluirse que la flexibilidad financiera es tanto más elevada cuanto:

a) mayor es el fondo de maniobra (grado de liquidez);

b) más rápido es el proceso de desmovilización de las inversiones (grado de movilidad);

c) mayor es la flexibilidad de las fuentes de financiación;

d) mayor es la capacidad de endeudamiento de la empresa (grado de elasticidad).

\begin{tabular}{|l|l|}
\hline \multicolumn{1}{|c|}{ Objetivo-vía } & \multicolumn{1}{c|}{ Medio } \\
\hline $\begin{array}{l}\text { 1. Reducción del capital invertido } \\
\text { (activo). }\end{array}$ & $\begin{array}{l}\text { - Externalización de actividades. } \\
\text { - Compartir recursos (cooperación empresarial). } \\
\text { - Mejor gestión del capital circulante. } \\
\text { - Recurso al alquiler:leasing, renting. }\end{array}$ \\
\hline $\begin{array}{l}\text { 2. Reducción del ratio de endeudamiento } \\
\text { (RA/RP). }\end{array}$ & $\begin{array}{l}\text { - Recurso a fondos propios (nuevos socios, financiación } \\
\text { bursátil -caso de empresas con cierta dimensión). }\end{array}$ \\
\hline 3. Inversiones más fácilmente desmovilizables. & - Lease back. \\
\hline $\begin{array}{l}\text { 4. Fuentes de financiaciónmás } \\
\text { flexibles. }\end{array}$ & $\begin{array}{l}\text { - Endeudamiento a corto plazo. } \\
\text { - Leasing y Renting. }\end{array}$ \\
\hline $\begin{array}{l}\text { 5. Diversificación de las fuentes de } \\
\text { financiación. }\end{array}$ & $\begin{array}{l}\text { - Aportación de capital de los socios. } \\
\text { la admisión a cotización y colocación deempréstitos en } \\
\text { otras plazas internacionales (reservado para grandes } \\
\text { empresas). }\end{array}$ \\
\hline $\begin{array}{l}\text { 6. Incremento del prestigio, la notoriedad } \\
\text { y la imagen pública de solvencia y } \\
\text { estabilidad. }\end{array}$ & $\begin{array}{l}\text { - Campañas publicitarias efectistas de carácter corporativo. } \\
\text { - Atención a la fecha de obligaciones de pago, } \\
\text { implementando adecuados sistemas de gestión de tesorería. }\end{array}$ \\
\hline
\end{tabular}

Figura 5 - Estrategias para el incremento de la flexibilidad financiera Fonte: Safón, 2000, p.183) 
En la figura 5 se recogen algunas estrategias (objetivos y medios) que pueden servir para incrementar la flexibilidad financiera de la empresa. Varias estrategias recogidas en esa figura están siendo empleadas por las empresas actuales con mayor o menor intensidad. La relación no pretende ser exhaustiva, sino más bien una muestra de cómo una organización puede ser flexibilizada desde el punto de vista financiero.

En relación con las estrategias y medios de la figura 5, el primer objetivo-vía, la reducción del activo, se produce reduciendo tanto el activo total como el pasivo exigible. Por ejemplo, si se mejora la gestión de los stocks, entonces se reduce la financiación a corto plazo de los almacenes; si se vende un edificio que se financió con un préstamo, entonces también se reduce el pasivo y el activo; si se mejoran los períodos de cobro y pago, a continuación puede ampliarse la diferencia entre el activo circulante y el pasivo circulante. Respecto a la cuarta vía, fuentes de financiación más flexibles, a medida que el endeudamiento es a más largo plazo, más inflexibilidad va añadiéndose a la estructura que, por regla general, no puede modificar a corto plazo ni la cantidad debida ni las condiciones del préstamo ${ }^{26}$. Igualmente, conforme las operaciones de financiación van siendo más autónomas respecto a las garantías empresariales, más elástica o dilatable va quedando la estructura financiera, por cuanto el potencial de crédito de la empresa no se ve reducido. Por otra parte, las fuentes de financiación flexibles proporcionan a la empresa un elevado grado de flexibilidad financiera, pero generan riesgos importantes, a saber: exigencia de mayores garantías, incremento del coste de la financiación debido a una coyuntura adversa, retirada de capital de algún socio, etc. Por último, cabe la contemplación del prestigio y la notoriedad de la empresa como fuente de flexibilidad financiera. En efecto, una empresa con un gran prestigio y una sólida imagen de solvencia financiera tiene mayores posibilidades de endeudamiento (préstamo, empréstito y/o ampliación de capital) a costes razonables, por lo que puede tener un margen mayor de actuación.

\subsection{LA FLEXIBILIDAD DEL FACTOR HUMANO}

La flexibilidad del factor humano, laboral o del trabajo, queda definida por:

a) la facultad de variar el número de asalariados;

b) la posibilidad de externalizar trabajos;

c) la facultad de incrementar el número de horas trabajadas sin variar la plantilla fija;

d) la posibilidad de usar el factor trabajo en actividades diferentes a las realizadas (polivalencia del trabajador);

e) la posibilidad de variar la remuneración del trabajador;

f) la posibilidad de desplazar geográficamente a los trabajadores.

La flexibilidad del factor humano depende, a nivel cuantitativo, de la capacidad de la

\footnotetext{
${ }^{26}$ No obstante, esto último no es exactamente cierto, o al menos no carece de cierta contradicción. Por ejemplo, un cambio de pasivo circulante a pasivo fijo incrementa el fondo de maniobra y paralelamente la flexibilidad financiera; pero ese cambio en la estructura financiera también añade inflexibilidad al pasivo de la empresa, debido a que se reduce la posibilidad de modificar a corto plazo las condiciones del endeudamiento. Se comprueba así que la medida propuesta tiene sus contraefectos, aunque el resultado neto sea el descrito en primera instancia. Desgraciadamente, la búsqueda de flexibilidad lleva en más ocasiones de las deseadas a disyuntivas y paradojas similares a la anterior; hecho que dificulta enormemente la medición de la flexibilidad financiera.
} 
empresa para adaptar y/o sustituir al factor humano. Normalmente, la primera opción, la adaptación del factor humano, implica formas organizativas basadas en la descentralización, la autonomía, el autocontrol, la interacción departamental, la formación continua de los recursos humanos y la creación de valores compartidos; elementos que permiten incrementar la competencia y el dominio de la complejidad presente en la actividad interna y externa de los trabajadores de la empresa ${ }^{27}$. La segunda opción, la sustitución del factor humano, depende del tipo de actividad que desarrolle la empresa y su posible grado de externalización (condiciones tecnológicas del ciclo de producción y carácter estratégico de las actividades), las restricciones del entorno jurídico-laboral, y las limitaciones del entorno económico y tecnológico. Respecto a esto último, para que una empresa pueda externalizar trabajos deben existir empresas que tengan los recursos y habilidades necesarios para realizar los trabajos subcontratados. En caso contrario, cuando en el entorno no existe el suficiente desarrollo industrial, la empresa no puede externalizar los trabajos por falta de empresas o especialistas mínimamente cualificados.

Desde los años setenta las empresas han utilizado dos grandes estrategias en pos de la flexibilización del factor humano: la estrategia de formación del personal y la concesión de mayor autonomía a los niveles operativos; y el recurso al trabajo flexible ${ }^{28}$. La primera de las dos grandes estrategias, la estrategia de formación del personal y la descentralización de la toma de decisiones y el control hacia los operarios, contribuye a extender nuevas formas de uso del personal y a crear supuestos de intercambiabilidad de los papeles como condiciones de flexibilidad del trabajo. Ejemplos de esta estrategia son los programas de formación del personal ${ }^{29}$, la rotación de puestos, el enriquecimiento del trabajo, el alargamiento del trabajo (job enlargement), y de forma más integrada, la involucración y capacitación del factor humano, así como el incremento de su poder y discrecionalidad (empowerment).

La segunda estrategia, la estrategia del trabajo flexible (temporal y externo), es la segunda vía de flexibilización observada en la práctica de las empresas actuales. Éstas, para eludir los vínculos de una relación "indefinida", y sobre todo para las actividades que no son estratégicas y/o esenciales, están recurriendo cada vez más al empleo de trabajo no estándar, flexible o temporal, y a la externalización de actividades para los niveles operativos y menos cualificados, así como al mercado de la consultoría para los niveles directivos. Algunos ejemplos de estas prácticas son los contratos de aprendizaje y formación, las becas, los trabajos a tiempo parcial, los trabajos eventuales y estacionales, el recurso a empresas de trabajo temporal (ETT) y la subcontratación de servicios (limpieza, mantenimiento de las instalaciones...); y, a nivel directivo, la consultoría y la asesoría en régimen temporal o externo.

Las prácticas de trabajo temporal y externo, necesarias para flexibilizar parte de las operaciones de la empresa, están afrontando una serie de problemas de implementación

\footnotetext{
${ }^{27}$ Este comentario se hace a nivel general, ya que no todas las empresas, departamentos ni tareas son iguales. En efecto, la gran heterogeneidad presente en la empresa: diversos niveles de complejidad en las tareas, subentornos distintos para departamentos distintos, diferentes tipos de recursos humanos (esenciales o periféricos), etc., obliga a relativizar cualquier afirmación de carácter general y simplista, como la realizada en el párrafo del texto principal.

${ }^{28}$ Respecto a la primera, existe evidencia empírica que demuestra la tendencia iniciada por las empresas en la década de 1970 hacia la multiprofesionalización de los trabajadores (por ejemplo: National Economic Development Office, 1986; Cross, 1988; y Whipp; Rosenfeld; Pettigrew (1989). En relación con la segunda, el recurso al trabajo flexible, algunas relevantes investigaciones pueden consultarse en National Economic Development Office (1986), Hakim (1990), Casey (1991), Marginson (1991), Penn (1992), O'Reilly (1992), Harrison y Kelley (1993), y Hunter ; McGregor; Maclnnes. (1993).

${ }^{29}$ Estos programas, ya sean realizados en el interior o el exterior de la empresa, consiguen que el factor humano sea más susceptible de cambios -en las estrategias y los modos de proceder- reduciéndose los riesgos de menores efectos en la curva de experiencia.
} 
que en ocasiones superan los beneficios pretendidos con la menor vinculación del factor trabajo. Estos problemas en el uso de trabajadores temporales o externos son la falta de coordinación, el desinterés, las tensiones internas, la falta de implicación y adaptación, y la dificultad en el control de la calidad de su trabajo. Son tan importantes los problemas que algunos autores dudan de las ventajas de esas prácticas. En este sentido, Albizu (1997) destaca que el trabajo no estándar -al margen de sus virtudes- presenta varios inconvenientes en su implementación:

a) crecimiento de los costes de producción y problemas con la capacidad de producción como resultado de las discontinuidades en las sustituciones, fallos de calidad, averías en las máquinas por un uso inexperto o negligente, retraso en los plazos de entrega, etc;

b) distorsiones en el grupo humano de la empresa debido a la alta rotación del personal no fijo;

c) aumento de los costes de reclutamiento, selección, acogida, integración y adiestramiento;

d) incremento de los costes de supervisión y control;

e) riesgo de abandono.

Geary (1992), a partir de un estudio empírico, también ha refrendado estos inconvenientes en el uso masivo de trabajo no estándar. Igualmente Blyton (1992) ha puesto en entredicho el trabajo flexible. En España, según el Instituto Sindical de Trabajo, Ambiente y Salud, los trabajadores eventuales tienen hasta cuatro veces más posibilidades de sufrir un accidente laboral que los empleados fijos.

En definitiva, el coste de implementación del trabajo flexible puede dejar sin efecto la mayor flexibilidad del trabajo conseguida merced al uso de trabajadores temporales y externos. Es por esto que la recomendación más razonable parece ser la búsqueda de la flexibilidad del trabajo mediante la labor formativa y la conservación de una fuerza laboral comprometida con el proyecto de la empresa y capacitada para adaptarse a cambios en sus conocimientos y habilidades.

\section{AUDIT DE LA FLEXIBILIDAD EN LAS PEQUEÑAS EMPRESAS}

El propósito del presente epígrafe es llegar a una propuesta de cuantificación de la flexibilidad empresarial. Por razones de simplicidad, el análisis va a diseñarse únicamente para las empresas de pequeña dimensión ${ }^{30}$, por lo que van a dejarse fuera del estudio a las medianas y las grandes empresas.

Dadas las características del caso que se pretende estudiar: la flexibilidad en las pequeñas empresas, se han determinado como únicas áreas de desarrollo en el cuestionario las correspondientes a la flexibilidad de los subsistemas funcionales principales y la flexibilidad del factor humano. Este proceder se justifica por la peculiar naturaleza de las empresas de pequeña dimensión en lo tocante a estrategia y estructura. En primer lugar, las estrategias de las pequeñas empresas suelen ser estrategias

\footnotetext{
${ }^{30}$ Sin entrar en la controversia de cuándo una empresa es micro, pequeña, mediana o grande, se utilizará el concepto de pequeña empresa haciendo alusión a las que, en un sector genérico, tienen una plantilla de entre 10 y 50 empleados.
} 
implícitas. En consecuencia, estas estrategias, que permanecen en los niveles tácitos de la empresa y en la mente del empresario/emprendedor, se manifiestan difícilmente observables y estudiables si no es a través de su manifestación mediante comportamientos, merced a las decisiones tomadas sobre las áreas funcionales y el factor humano. De esta manera, ante la dificultad para analizar y estudiar el grado de modificación y sustitución de las estrategias de las pequeñas empresas, se ha pensado que existe una natural cristalización de éstas en las áreas funcionales y en la forma de gestión del factor humano. Así, al analizar estas formas de gestión podrá verse también el grado de flexibilidad estratégica.

En segundo lugar, la estructura organizativa de las pequeñas empresas corresponde a diseños estructurales simples e informales, y en consecuencia, caracterizados por una gran flexibilidad en las relaciones y comunicaciones. Así pues, ante la dificultad para identificar grados de flexibilidad diferentes en estructuras tan básicas, y con el convencimiento de que, al igual que ocurre con la flexibilidad de la estrategia, la flexibilidad de los subsistemas funcionales y del factor humano está condicionada por la flexibilidad de la estructura, se piensa que, al plantear las preguntas del cuestionario relativas a las áreas funcionales y el factor humano, el empresario consultado está respondiendo también a la flexibilidad de la estrategia y la flexibilidad de la estructura. Es más, igualmente se está seguro de que, ante las preguntas formuladas, el sujeto interrogado es capaz de hacer frente al problema no sólo analizando un elemento concreto, sino al mismo tiempo observando el elemento en sí, las relaciones con los otros elementos (póngase por caso producción y factor humano), y las relaciones con el todo o la empresa en su conjunto. Por todo lo dicho, se entiende que practicando un análisis sobre la flexibilidad de los subsistemas funcionales y del factor humano de la empresa, se llega a obtener una medida de la flexibilidad estratégica y estructural.

Ahora bien, debe tenerse en cuenta que el cuestionario va a diseñarse con un objetivo claro y concreto: identificar la flexibilidad de las pequeñas empresas. Por consiguiente, este cuestionario es un instrumento únicamente válido para el estudio de empresas de pequeña dimensión, dada su evidente sencillez. En el caso de empresas de mayor dimensión, el cuestionario debería ampliarse desarrollando explícitamente las flexibilidades de la estrategia y la estructura -sin olvidar la de los recursos, aunque una parte de ésta se estudia al tratar el factor humano-, para recoger las nuevas posibilidades que ofrecen las empresas más complejas con diseños de estructura más sofisticados, y procesos de formulación e implementación de estrategia explícitos.

Resumiendo, el cuestionario que va a presentarse es una herramienta para el estudio de la pequeña empresa, y está construido ad hoc para los propósitos ilustrativos del presente análisis. Debe, por ello, entenderse como una primera aproximación cuantitativa al fenómeno de la flexibilidad de la empresa.

Seguidamente se presenta el cuestionario articulado en cuatro secciones: flexibilidad comercial, flexibilidad productiva, flexibilidad financiera y flexibilidad del factor humano.

\subsection{CUESTIONES PREVIAS AL CUESTIONARIO}

Antes de abordar las preguntas del cuestionario conviene plantear unas consideraciones previas para su uso e interpretación. En la batería de preguntas que se recogen a continuación hay sistemáticas referencias a los tiempos de respuesta. Éstos se han clasificado en cuatro horizontes: respuesta instantánea (una semana), respuesta a corto plazo (un mes), respuesta a medio plazo (un año) y respuesta a largo plazo (tres 
años). Evidentemente los horizontes propuestos pueden modificarse arbitrariamente, tanto en el número de escenarios como en su separación temporal, si el caso o sector tratado lo requieren.

En segundo lugar, aunque más adelante vamos a realizar una simulación del cuestionario de tipo transversal (varias empresas en un momento del tiempo), también puede usarse en análisis longitudinales, es decir, análisis para una única empresa en distintos momentos del tiempo. En ambos casos, el investigador debe contemplar siempre los mismos acontecimientos para todas las empresas consultadas (análisis transversal) o para las diferentes consultas en la misma empresa (análisis longitudinal), ya que, como consecuencia de la ecuación del grado de flexibilidad de la empresa, sólo a iguales procesos de cambio es posible comparar el grado de flexibilidad de diferentes empresas o de diferentes momentos de la misma empresa.

A continuación se muestran las preguntas del cuestionario.

\subsection{CUESTIONARIO}

\section{FLEXIBILIDAD COMERCIAL}

\subsection{Flexibilidad de la oferta comercial actual}

$\mathrm{P} 1 \mathrm{a}^{31}$. Número de líneas de productos diferentes ${ }^{32}$ que se ofertan.

P1a'. Concentración de volúmenes ${ }^{33}$.

P1b. Número de líneas de productos independientes que se ofertan.

P1b'. Concentración de volúmenes.

P2. Número de clientes vivos (activos) independientes.

P2'. Número de clientes que concentran el x\% de la facturación (por ejemplo, el 80\%).

\subsection{Flexibilidad en la introducción de nuevos productos ${ }^{34}$}

P3. ¿Cuánto tiempo se tarda como media en lanzar un nuevo producto desde que se concibe su necesidad?

\subsection{Flexibilidad de los precios}

$\mathrm{P} 4-\mathrm{P} 7^{35}$. Si fuera preciso cambiar los precios para que surtieran efecto en [una semana, un mes, un año, tres años] (por ejemplo, por un previsible cambio en la paridad de las

\footnotetext{
${ }^{31}$ Se abrevia "pregunta" con "P".

${ }^{32} \mathrm{El}$ grado de diferencia debe ser definido por el investigador. No se exige, en esta pregunta, que las líneas de productos sean independientes.

${ }^{33}$ Las preguntas P1a' y P1b' van acompañadas de estudios de concentración de volúmenes, entendiendo un mayor grado de flexibilidad conforme se reduce la concentración. Para el estudio de la concentración puede usarse el índice de Herfindahl. En la representación gráfica de esas variables se practicará una transformación del tipo a'=1-a para darles el sentido del signo de la flexibilidad de las demás preguntas.

${ }^{34} \mathrm{El}$ resto de la flexibilidad de producto se verá en la sección del cuestionario dedicada a la flexibilidad productiva.

${ }^{35} \mathrm{En}$ la lectura de este grupo de preguntas y de los grupos posteriores deben tenerse presentes dos cosas: a) "P4-P7" significa preguntas 4 a 7 , y por tanto, preguntas $4,5,6$ y 7 ; y b) cada pregunta debe ser leída utilizando el enunciado con normalidad, pero teniendo la precaución de leer sólo el término que, situado entre corchetes y separado por comas, coincide con su orden dentro del grupo de preguntas: pregunta 4 con "una semana", pregunta 5 con "un mes", pregunta 6 con "un año" y pregunta 7 con "tres años", y así para todos los grupos de preguntas de este cuestionario, que se ha presentado de esta forma para no sobrecargarlo con redundancias.
} 
divisas): ¿sería posible modificarlos?; ¿sería capaz de comunicárselo a sus clientes actuales y potenciales de una manera económica? Responda en cada caso el porcentaje de clientes avisados.

\subsection{Flexibilidad de la distribución}

P8-P11. ¿Podría distribuir sus productos a través de canales diferentes a los que actualmente utiliza, si este requerimiento se le manifestara en [una semana, un mes, un año, tres años], en condiciones de costes razonables? Responda en cada caso como porcentaje de lo actualmente distribuido.

\subsection{Flexibilidad de la comunicación}

P12-P15. ¿Sería capaz de modificar o incluso sustituir de manera económica las actuales formas de comunicación (publicidad y fuerza de ventas fundamentalmente), si fuera necesario ante un acontecimiento que se previera para dentro de [una semana, un mes, un año, tres años]? Responda en cada caso como porcentaje de los actuales medios.

\section{FLEXIBILIDAD PRODUCTIVA}

\subsection{Flexibilidad de los volúmenes de producción}

P16-P19. ¿Qué incremento de producción máximo podría acometer si la demanda se incrementara en un $\mathrm{x} \%$ (por ejemplo, un $500 \%$ ) para dentro de [una semana, un mes, un año, tres años] en los productos que más factura?

P20-P23. ¿Cuál sería el incremento estimado de coste por producto, en el caso de hacer frente al incremento en el espacio de [una semana, un mes, un año, tres años]?

P24-P27. ¿Qué reducción de producción máxima podría acometer si la demanda se redujera en un $\mathrm{x} \%$ (por ejemplo, un 90\%) para dentro de [una semana, un mes, un año, tres años] en los productos que más factura?

P28-P31. ¿Cuál sería el incremento de coste por producto estimado en el caso de hacer frente a la reducción en el espacio de [una semana, un mes, un año, tres años]?

\subsection{Flexibilidad de las tipologías (variedad de productos)}

P32-P35. ¿Cuántos tipos diferentes (aunque sean diferencias mínimas) de productos sería capaz de producir si así lo exigiera el mercado para dentro de [una semana, un mes, un año, tres años]? Piense como pedido mínimo un promedio de los que actualmente sirve y que las exigencias no obligan a producir todas las posibles variaciones al mismo tiempo. Realice el análisis individualmente.

P36-P39. ¿Serían sus productos "nuevos" competitivos (desde el punto de vista de los costes de producción) en el caso de los producidos para dentro de [una semana, un mes, un año, tres años]?

\subsection{Flexibilidad de las calidades}

P40-P43. ¿Cuántos niveles de calidad diferentes puede aplicar a sus productos si las exigencias de la demanda se producen en [una semana, un mes, un año, tres años]? Piense en calidades reconocidas por los clientes y genéricas para el mercado. 
P44-P47. ¿Cuál sería el incremento de coste por producto estimado en los diferentes casos ([una semana, un mes, un año, tres años])? Ofrezca una medida en porcentaje respecto al coste anterior.

\subsection{Flexibilidad de las fuentes de aprovisionamiento}

P48-P51. Si fuera necesario cambiar de proveedores en [una semana, un mes, un año, tres años], ¿podría hacerlo sin retrasos en los períodos de entrega, mermas en la calidad ni incrementos en los costes (por precios, por necesidad de crear nuevos contactos, por el transporte...)? Piense en los proveedores de sus inputs fundamentales por su importancia relativa o exclusividad (dificultad en la sustitución). Responda como porcentaje del volumen actual de compras a proveedores.

P52. Número de proveedores vivos (activos) afectos a producción.

P52'. Número de proveedores vivos que concentran el $80 \%$ de las compras a proveedores.

\section{FLEXIBILIDAD FINANCIERA}

P53. Ratio actual de capital circulante o fondo de maniobra.

P54. Activo fijo realizable instantáneamente sin comprometer el negocio (Activo fijo realizable actualizado/Activo total real actualizado).

P55. Potencial de crédito (Activo total real actualizado/Pasivo exigible).

$\mathrm{P} 56-\mathrm{P} 59^{36}$. Ante un acontecimiento imprevisto (oportunidad o amenaza) que requiere actuaciones para dentro de [una semana, un mes, un año, tres años], ¿con cuánto dinero (recursos financieros) se encontraría (grado de liquidez-movilidad) y cuánto podría pedir prestado (grado de elasticidad), todo ello a un coste razonable (por ejemplo, no más allá de dos puntos por encima de su actual coste de capital)?

\section{FLEXIBILIDAD DEL TRABAJO}

\subsection{Flexibilidad de la plantilla o grado de vinculación del factor trabajo a la empresa}

P60.

$N^{0}$ de horas/semana trabajadas por trabajadores temporales (no fijos)

$\mathrm{N}^{\circ}$ total de horas/semana trabajadas

\subsection{Flexibilidad numérica y financiera}

Ante un [incremento, decremento] de un $\mathrm{x} \%$ (por ejemplo: [500\%, 90\%]) de los pedidos para dentro de [una semana, un mes, un año, tres años]:

\footnotetext{
${ }^{36}$ Las respuestas a las preguntas 56 a 59 se ofrecen como porcentaje del activo total real actualizado.
} 
P61-P68. ¿Podría [contratar, despedir] las necesarias horas/hombre (cualificadas y no cualificadas) de forma económica? Conteste como porcentaje del total necesitadas, es decir, el porcentaje de aquéllas que sí logra [contratar, despedir].

P69-P76. ¿Podría [incrementar, reducir] los salarios de los trabajadores fijos? Conteste como porcentaje de las horas/hombre actualmente trabajadas.

P77-P84. ¿Podría [aumentar, reducir] las horas trabajadas por los trabajadores fijos sin dejar de trabajar eficientemente?

P85-P88. Ante una huelga anunciada para dentro de [una semana, un mes, un año, tres años] de una duración de por ejemplo diez días, ¿qué porcentaje de la producción podría presentar para hacer frente a los pedidos habituales? Piense en las posibilidades de contratar trabajo temporal (apto) o de recurrir a compras a sus competidores.

\subsection{Flexibilidad funcional}

P89-P92. Ante nuevas exigencias de los clientes que se manifestarán dentro de [una semana, un mes, un año, tres años], ¿podrían sus empleados cambiar de puesto de trabajo y seguir siendo eficientes (con el necesario rodaje) por contar con los conocimientos pertinentes? Responda pensando en el porcentaje de empleados capacitados sobre el total, y sobre una escala de 1 a 10 sobre la capacidad de adaptación de éstos. (Esta pregunta tiene dos respuestas para cada horizonte temporal, aunque se traducirán en una dimensión multiplicando las dos variables consideradas: porcentaje y valoración).

\subsection{Flexibilidad geográfica}

P93-P96. ¿Qué porcentaje de sus trabajadores estarían dispuestos a cambiar de residencia sin exigir compensaciones excesivamente costosas?, ¿y de sus vendedores?, ¿y de sus mandos intermedios?, ¿y de sus directores de departamentos?

\section{Perfiles flexibles}

El objetivo de este apartado es ilustrar una experiencia práctica de la aplicación del cuestionario en lo que podría llamarse el audit de la flexibilidad de una empresa. A este efecto se ha diseñado una prueba ficticia basada en una simulación de empresas, contemplando y confrontando dos empresas manufactureras de tamaño medio, con un solo negocio y de un sector industrial tradicional (empresas A y B). Los resultados simulados se han ilustrado gráficamente, mostrando unos perfiles flexibles multidimensionales para la contemplación de la flexibilidad en las empresas de manera gráfica. Para la ilustración de estos perfiles se han simulado las respuestas a las preguntas del cuestionario (series correspondientes al título datos obtenidos), y a continuación se han normalizado los datos en unas series transformadas. A la hora de analizar las series transformadas hay que tener en cuenta la salvedad de que, en los casos en los que la respuesta representa una pérdida de flexibilidad, se recoge la transformación $z^{\prime}=1-z$ para facilitar la lectura gráfica en términos de flexibilidad. Así, por ejemplo, si se toma la pregunta 1a', Concentración de volúmenes, el valor real obtenido para la empresa A ha sido de 0,7 y para la empresa $B$ de 1 . Estos valores, que reflejan una mayor flexibilidad conforme se acercan a cero, han sido transformados para que la flexibilidad siempre se exprese como crecimiento en las magnitudes tratadas, de forma que se tengan todas las variables homogeneizadas en su comportamiento. Las series transformadas se realizan 
merced a una conversión que asigna el valor de la unidad a las variables de la empresa $B$, y el cociente entre las variables de las empresas $A$ y $B$ a las variables de la empresa $A$; de manera que se observe su presencia en términos relativos frente a las variables de la empresa B. Por ejemplo, la pregunta 2', Número de clientes que concentran el $80 \%$ de la facturación, presenta unos resultados de 20 para la empresa A y 3 para la empresa B. Esto significa que en esa variable concreta el caso A se manifiesta más flexible que el caso B. Ahora bien, para ilustrarlos gráficamente, debido a la dificultad para mostrar homogéneamente un fenómeno multidimensional, se ha normalizado la escala, asignando a la serie de la empresa B el valor de la unidad (en el ejemplo haciendo la transformación de 3 a 1), y a la serie de la empresa A el cociente entre su valor original y el de la empresa B (en el ejemplo haciendo el cociente $20 / 3$ que resulta 6,67). En suma, las series transformadas permiten trabajar en términos de porcentaje (si se multiplica por cien) y con valores homogéneos (con la misma unidad y escala).

\section{DATOS OBTENIDOS}

1. Flexibilidad comercial

\begin{tabular}{|l|r|r|}
\hline Preg. & Emp. A & \multicolumn{1}{|c|}{ Emp. B } \\
\hline P1a & 2 & 1 \\
\hline P1a' & 0,7 & 1 \\
\hline P1b & 0 & 0 \\
\hline P1b' $^{\prime}$ & 1 & 1 \\
\hline P2 & 100 & 70 \\
\hline P2' & 20 & 3 \\
\hline P3 & 0,25 & 0,75 \\
\hline P4-7 & 1 & 0,5 \\
\hline P8-11 & 0,6 & 0,25 \\
\hline P12-15 & 0,1 & 0 \\
\hline
\end{tabular}

2. Flexibilidad productiva

\begin{tabular}{|l|r|r|}
\hline Preg. & Emp. A & Emp. B \\
\hline P16-19 & 2 & 4 \\
\hline P20-23 & 0,15 & 0,1 \\
\hline P24-27 & 0,7 & 0,4 \\
\hline P28-31 & 0,4 & 0,5 \\
\hline P32-35 & 10 & 0 \\
\hline P36-39 & SI & NO \\
\hline P40-43 & 4 & 2 \\
\hline P44-47 & 0 & 0,1 \\
\hline P48-51 & 0,5 & 0,2 \\
\hline P52 & 50 & 20 \\
\hline P52' & 10 & 4 \\
\hline
\end{tabular}

3. Flexibilidad financiera

\begin{tabular}{|l|r|r|}
\hline Preg. & Emp. A & \multicolumn{1}{|c|}{ Emp. B } \\
\hline P53 & 1,3 & 1,2 \\
\hline P54 & 0 & 0 \\
\hline P55 & 3 & 2,8 \\
\hline P56-59 & 0,3 & 0,25 \\
\hline
\end{tabular}

4. Flexibilidad del trabajo

\begin{tabular}{|l|r|r|}
\hline Preg. & Emp. A & Emp. B \\
\hline P60 & 0,1 & 0,25 \\
\hline P61-64 & 0,75 & 0,75 \\
\hline P65-68 & 0 & 0 \\
\hline P69-72 & SI & SI \\
\hline P73-76 & NO & NO \\
\hline P77-80 & SI & SI \\
\hline P81-84 & SI & SI \\
\hline P85-88 & 1 & 1 \\
\hline P89-92 & 2,75 & 2,05 \\
\hline P93 & 0,1 & 0,1 \\
\hline P94 & 0,3 & 0,3 \\
\hline P95 & 0,1 & 0,1 \\
\hline P96 & 0,2 & 0,2 \\
\hline
\end{tabular}

La ilustración de los resultados se realiza a partir de las series transformadas y unos gráficos radiales. Estos gráficos, que forman los perfiles flexibles, se han propuesto para permitir una visualización rápida del fenómeno, ya que denuncian las asimetrías de las dos empresas analizadas a nivel de flexibilidad y desde un punto de vista relativo. 
Como quiera que el objetivo de este apartado es puramente didáctico, en beneficio de la claridad se han agrupado las preguntas en una respuesta única correspondiente al plazo de un mes, en lugar de desdoblar el cuestionario en los cuatro horizontes temporales previstos.

Seguidamente se da paso a la reproducción de los resultados simulados, las series transformadas y los gráficos que ilustran los perfiles flexibles.

Con fines exclusivamente ilustrativos va a realizarse un breve comentario del ejercicio simulado. Comenzando por el análisis de la flexibilidad comercial (figura 6), a la vista de los resultados puede afirmarse que han sido observadas diferencias significativas entre las prestaciones flexibles de las empresas A y B.

La empresa A muestra el ejemplo de una empresa con dos líneas de productos relacionadas, que podrían diferenciarse por su nivel de calidad y presentación. La empresa $B$ recoge el caso de una empresa con una oferta comercial reducida que comercializa sólo un tipo de producto. En esta primera variable, amplitud de la gama, la empresa A es más flexible que la B, como lo muestran las preguntas 1a y 1a'. Sin embargo, no existen diferencias tras el análisis de las relaciones entre líneas de producto (preguntas $1 \mathrm{~b}$ y $1 b^{\prime}$ ).

\section{SERIES TRANSFORMADAS}

1. Flexibilidad comercial

\begin{tabular}{|l|r|r|}
\hline \multicolumn{1}{|c|}{ Preg. } & Emp. A & Emp. B \\
\hline P1a & 2,00 & 1 \\
\hline P1a' $^{\prime}$ & 30,00 & 1 \\
\hline P1b & 1 & 1 \\
\hline P1b' & 1 & 1 \\
\hline P2 & 1,43 & 1 \\
\hline P2' & 6,67 & 1 \\
\hline P3 & 3,00 & 1 \\
\hline P4-7 & 2,00 & 1 \\
\hline P8-11 & 2,40 & 1 \\
\hline P12-15 & 1 & 1 \\
\hline
\end{tabular}

2. Flexibilidad productiva

\begin{tabular}{|l|r|r|}
\hline Preg. & Emp. A & Emp. B \\
\hline P16-19 & 0,50 & 1 \\
\hline P20-23 & 0,94 & 1 \\
\hline P24-27 & 1,75 & 1 \\
\hline P28-31 & 1,20 & 1 \\
\hline P32-35 & 100,00 & 1 \\
\hline P36-39 & 2,00 & 1 \\
\hline P40-43 & 2,00 & 1 \\
\hline P44-47 & 1,11 & 1 \\
\hline P48-51 & 2,50 & 1 \\
\hline P52 & 2,50 & 1 \\
\hline P52 & 2,50 & 1 \\
\hline
\end{tabular}

3. Flexibilidad financiera

\begin{tabular}{|l|r|r|}
\hline Preg. & Emp. A & Emp. B \\
\hline P53 & 1,08 & 1 \\
\hline P54 & 1 & 1 \\
\hline P55 & 1,07 & 1 \\
\hline P56-59 & 1,20 & 1 \\
\hline
\end{tabular}

4. Flexibilidad del trabajo

\begin{tabular}{|l|r|r|}
\hline \multicolumn{1}{|c|}{ Preg. } & Emp. A & Emp. B \\
\hline P60 & 0,40 & 1 \\
\hline P61-64 & 1,00 & 1 \\
\hline P65-68 & 1 & 1 \\
\hline P69-72 & 1 & 1 \\
\hline P73-76 & 1 & 1 \\
\hline P77-80 & 1 & 1 \\
\hline P81-84 & 1 & 1 \\
\hline P85-88 & 1,00 & 1 \\
\hline P89-92 & 1,34 & 1 \\
\hline P93 & 1,00 & 1 \\
\hline P94 & 1,00 & 1 \\
\hline P95 & 1,00 & 1 \\
\hline P96 & 1,00 & 1 \\
\hline
\end{tabular}


La empresa $A$ presenta una cartera de clientes numerosa y concentra sus ventas en veinte de ellos. La empresa $B$, en cambio, sirve a menos clientes y concentra su producción únicamente en tres grandes clientes, determinando así una mayor inflexibilidad que A (preguntas 2 y $2^{\prime}$ ).

En la velocidad de introducción de productos la empresa A coordina mejor sus funciones de marketing, producción e I+D, y consigue ser el triple mejor que su homóloga B, con problemas de integración de funciones (pregunta 3).

El cambio de precios está condicionado por diversas variables. La empresa A tiene una red comercial más numerosa que la empresa $B$ y, comparativamente, tiene mayor poder sobre sus clientes. Debido a esto la empresa $A$ ofrece mejores resultados en la variable correspondiente (pregunta 4-7).

En el caso de la distribución ambas empresas facturan directamente a sus clientes, que son en todos los casos consumidores finales. El transporte y almacenamiento fuera de la fábrica es costeado por los clientes de manera habitual. La empresa A está ubicada en una zona industrial con abundancia de recursos del tipo requerido y con un número elevado de compradores de su producto, que puede catalogarse como de estándar para el mercado. La empresa B se halla en una situación distinta: tiene acuerdos contractuales con sus tres principales clientes y se encuentra en una zona industrial con escasez de recursos relacionados con la logística externa. El resultado de la relación menos vinculante y la abundancia de recursos y compradores próximos hacen que la empresa A exhiba una mejor puntuación en la pregunta 8-11. Las dos empresas "llegan" a sus clientes utilizando la fuerza de ventas ${ }^{37}$. La empresa $A$ tiene más vendedores que la empresa $B$, pero, sin embargo, sólo tiene una pequeña porción en nómina y de carácter fijo, ya que el resto son agentes comerciales independientes que trabajan a comisión. La empresa B tiene menos vendedores y son todos fijos. Las dos presentan similares cualificaciones en los vendedores fijos. Confrontándolas a la pregunta 12-15 las dos se muestran muy rígidas. La empresa A no puede llegar a acuerdos con nuevos comisionistas en el plazo de un mes. Tampoco puede conseguir vendedores que trabajen eficientemente en ese plazo. Todo esto se reproduce también en el caso de la empresa B. Sin embargo, debido a la mayor versatilidad de los operarios de la empresa A, ésta puede proponer soluciones transitorias utilizando "vendedores" de su propia organización y conseguir una respuesta ligeramente superior a B. Además, la empresa A también puede considerar la opción de vender a través de otros competidores de su zona; y la de contratar, de manera más fácil que $B$, vendedores de la competencia que se radican en la misma zona.

\footnotetext{
${ }^{37} \mathrm{La}$ pregunta correspondiente a la flexibilidad de la comunicación va a ser respuesta en función de la posibilidad de cambiar la fuerza de ventas. Sin embargo, en un análisis más riguroso puede contemplarse un caso de sustitución de la forma en la que la empresa "llega" e informa a sus clientes.
} 

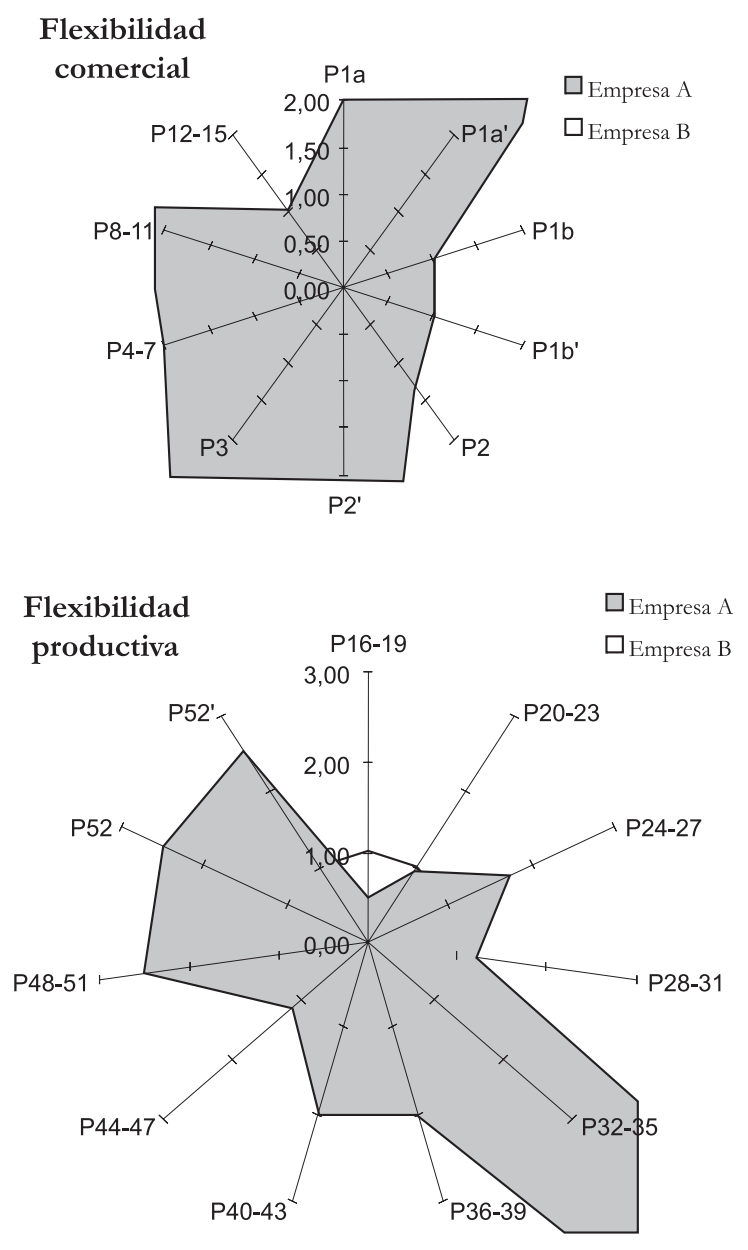

Figuras 6 y 7 - Representación gráfica de los perfiles flexibles

Desde el punto de vista de los costes, en el caso de un crecimiento importante de la producción en ambas empresas se aumentan los costes de la mano de obra (horas extra, nuevos contratos, trabajo temporal...), el mantenimiento, las amortizaciones, el control, etc.; y se reducen los costes de estructura imputados a cada unidad de producto. El efecto neto no evidencia diferencias entre las dos empresas, sin embargo, merece la pena destacar que la empresa B centra su atención en sus actividades internas (costes internos), y la empresa A lo hace especialmente en los costes transmitidos por su red de subcontratistas. Así, por ejemplo, mientras la empresa B trabaja para los almacenes durante el tiempo anterior al pedido y soporta un coste de oportunidad por el capital invertido, la empresa A puede mantener este coste en los subcontratistas, según se lo posibilite o no su sistema de producción y de relación con los mismos.

La flexibilidad de los volúmenes de producción cuando la demanda se contrae espectacularmente pone a la empresa ante el problema de saber cómo resolver la cuestión de los costes fijos (tributos, alquileres, amortizaciones, salarios fijos...) y la liquidación o reconversión de los activos. Este acontecimiento pone en jaque a cualquier empresa, 
dependiendo su respuesta en gran medida de cómo se realice la producción: toda dentro de la empresa, o parte dentro y parte fuera (subcontratación). También depende de si la reducción de la demanda es coyuntural o permanente. En el primer caso la empresa puede obviar parcialmente las circunstancias adversas y trabajar para los almacenes, que, pasada la contingencia, irán reduciéndose. Esto, unido a una leve bajada del ritmo de producción, debería ser suficiente. En el segundo caso la empresa debe pensar en su diversificación, reconversión o liquidación, aunque queda siempre la posibilidad de ser una de las pocas empresas que se queden en el mercado ganando lo dejado por las menos eficientes.

Tratando el problema anterior como estructural, la empresa A se encuentra mejor preparada para cambiar de productos y reformular su negocio buscando nuevos mercados en crecimiento. Además, cuenta con la posibilidad de rescindir los contratos de subcontratación y reducir su capacidad productiva sin afrontar una reestructuración como la necesitada en B (pregunta 24-27). Por el lado de los costes la empresa B soporta peor la reducción de la producción debido a su estructura de costes fijos, y la especialización y dedicación de sus activos (pregunta 28-31). La flexibilidad de las tipologías (variedad de productos) es claramente favorable a la empresa A (preguntas 32-35 y 36-39). Lo mismo ocurre con las calidades (preguntas 40-43 y 44-47). En ambos casos, tipologías y calidades, la empresa A, de acuerdo con las hipótesis productivas de cada empresa, se manifiesta más flexible que su homóloga $B$.

La empresa A se halla en una zona industrial con una fuerte especialización y concentración productiva, con abundancia de fabricantes, proveedores, clientes y servicios (financieros, de transporte...), y con relaciones de subcontratación muy desarrolladas. Por el contrario, la empresa B se localiza en una zona agraria sin especialización industrial y con escasez de recursos complementarios. El análisis anterior da como resultado un mejor comportamiento de la empresa A en relación con las fuentes de aprovisionamiento, al margen de que también mantenga una cartera de proveedores más extensa (preguntas 48-51, 52 y $52 ')$.

El análisis de la flexibilidad del sistema de producción se ilustra en la figura 7 y parte de las siguientes premisas:

\begin{tabular}{|l|c|c|}
\hline \multicolumn{1}{|c|}{ Dimensión } & $\begin{array}{c}\text { Empresa } \\
\text { A }\end{array}$ & $\begin{array}{c}\text { Empres } \\
\text { a B }\end{array}$ \\
\hline Nivel de especialización de la fábrica & Bajo & Alto \\
\hline Diferenciación de la producción & Alta & Nula \\
\hline Tamaño de los lotes & Pequeño & Grande \\
\hline Cualificación de los obreros & Alta & Baja \\
\hline Polivalencia de los obreros & Alta & Baja \\
\hline Subcontratación de capacidad & Alta & Nula \\
\hline Subcontratación de especialidad & Alta & Baja \\
\hline
\end{tabular}

Las asunciones anteriores definen una empresa $A$ basada en criterios de especialización flexible y una empresa B fundada en la filosofía de la producción en masa. Ambos casos permiten dicotomizar dos modos de producir extremos y evidenciar sus virtudes y defectos. La empresa B es más potente que la empresa A en lo tocante a la flexibilidad de los incrementos de los volúmenes de producción (pregunta 16-19). Esta empresa puede contratar más fácilmente personal para incrementar los turnos y responder así al incremento de la demanda. En el lado contrario se encuentra la empresa A, que debe invertir más tiempo en la formación de sus trabajadores, aun cuando se aprovecha 
de todas las posibilidades que le ofrece la subcontratación. Cabe también, para las dos empresas, el recurso a los almacenes, que según el plazo que se tenga para afrontar las demandas futuras pueden utilizarse para amortiguar sus efectos desestabilizadores.

El área financiera es el capítulo con menos contrastes (figura 8). La empresa B ha realizado en los últimos tiempos una fuerte inversión en la automatización y robotización de su planta, ocasionando algunos problemas de liquidez, aunque se han cubierto con un préstamo a medio plazo. La empresa espera crecer sin tener que contratar mano de obra de taller, si bien estos beneficios se recogerán conforme vayan rentabilizándose las inversiones y crezcan los ratios de productividad del capital y el trabajo. Además, la empresa $B$ tiene un período medio de maduración superior a la empresa A, derivado de su peor posición frente a proveedores y clientes, aunque respecto a otras variables se acerca mucho a la empresa A e incluso la mejora. En cualquier caso, globalmente el ciclo es ligeramente superior.

La empresa A goza de un balance con menos activo y pasivo total que la empresa B. Esto es debido a que se "apalanca" en sus subcontratistas y a que ha externalizado gran parte de las operaciones que no son vistas como fundamentales. Por ejemplo, destaca el uso en régimen de alquiler de ciertas máquinas de propósito universal, arrendadas a una empresa de leasing. También es notoria la imagen y el prestigio de la empresa $A$, que durante varias décadas ha sido ejemplo de buena gestión.

Continuando con la simulación, la empresa A presenta un capital circulante mayor, aunque no muy lejos del de B (pregunta 53). Tanto A como B se hallan utilizando todos sus recursos, por lo que no pueden responder en ninguna medida a la opción planteada en la pregunta 54. En relación con el potencial de crédito tampoco caben diferencias significativas; sin embargo, la empresa A puntúa tenuemente mejor que la empresa B, merced a una valoración más positiva de su activo, que tiene un valor de mercado más elevado por ser menos especializado y estar la empresa A menos apalancada financieramente (pregunta 55). Ante un requerimiento de fondos ocasionado por un acontecimiento imprevisto, ambas empresas responden de manera similar; empero, la reputación de la empresa A logra que mejore los fondos captados y, en comparación con su menor activo, el ratio de la pregunta 56-59 supera tímidamente al de su homóloga B. En síntesis, la valoración global del área financiera muestra que la empresa A es igual o ligeramente mejor que la empresa $\mathrm{B}$.

La última flexibilidad del análisis es la flexibilidad del trabajo (figura 9). La empresa A cuenta con unos trabajadores de fábrica más cualificados que la empresa B y se encuentra en una zona industrial con gran abundancia de recursos humanos cualificados, debido a la tradición histórica y la concentración de empresas de su ramo. Como consecuencia del tipo de sistema productivo de las empresas A y B, la segunda tiene más posibilidad de contratar trabajo flexible (básicamente temporal), siendo mejor evaluada globalmente en la pregunta 60 . No obstante, si el análisis se hiciera por departamentos, la empresa A puntuaría mejor, por ejemplo, en los empleados afectos a marketing. 

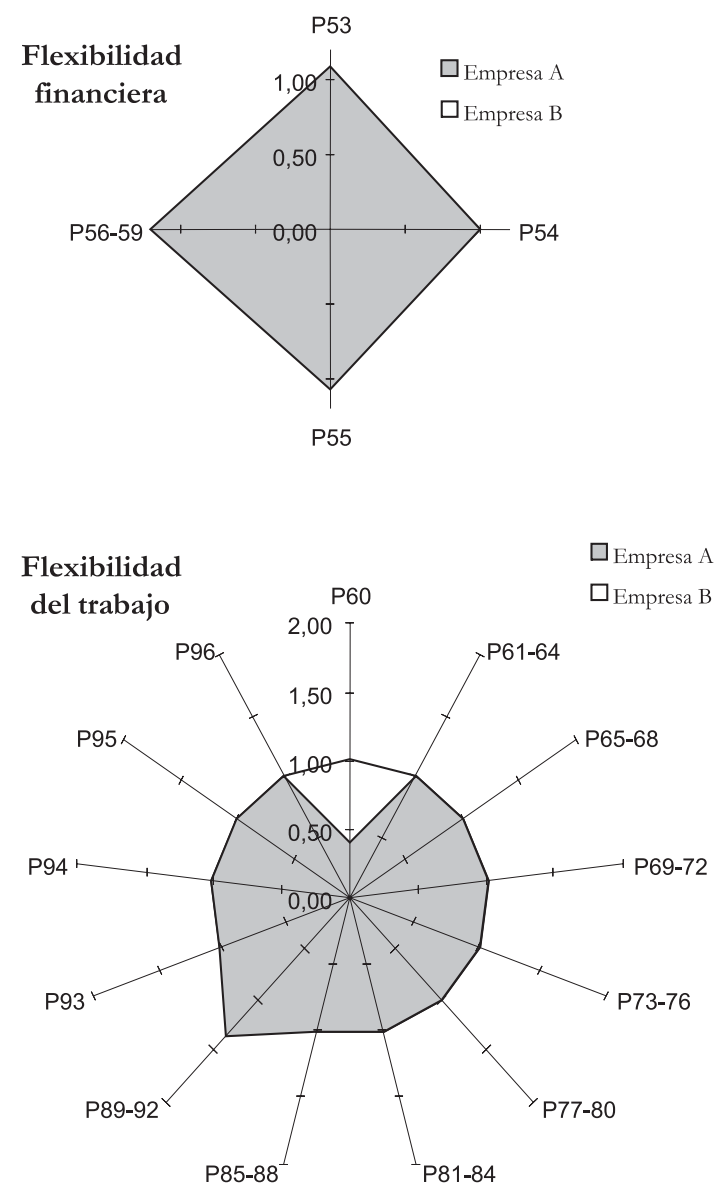

Figuras 8 y 9 Representación gráfica de los perfiles flexibles

Cada empresa afronta el crecimiento de manera diferente. La empresa B busca cubrir todo el incremento de demanda de forma interna, de manera que se rentabilicen al máximo sus inversiones en capital. Este crecimiento le supone una fuerte necesidad de horas/hombre, para evitar las paradas y cubrir todos los turnos posibles, sobre todo en los cuellos de botella. La empresa B tiene la ventaja del poco nivel de cualificación que exige a la mayoría de sus obreros y el poder llegar a una explotación de las máquinas de 168 horas/máquina semanales, con tiempos mínimos en mantenimiento y nulos en preparación. En el extremo opuesto se halla la empresa A. Ésta acomete el crecimiento de una manera mixta: vía externa e interna. Esta forma de buscar el volumen le confiere la ventaja de requerir menos inversión en planta y menos mano de obra para acometer el crecimiento, pero a cambio le obliga a mantener costes de coordinación con su red de subcontratistas. La empresa A posee además el inconveniente de contar con obreros muy cualificados y mejor remunerados que $B$, si bien presenta la ventaja de hallarse en una zona donde este recurso abunda.

Por cuanto se ha dicho, el resultado de la pregunta 61-64, alcanzado de manera muy diversa, es similar en ambos casos, y puede concluirse que cada empresa, según su personalidad, se adapta de una forma distinta. 
La flexibilidad del despido viene condicionada por la falta de flexibilidad del ciclo productivo. Este hecho es muy importante en la empresa $B$, que tiene un mínimo de operarios por debajo del cual se paraliza la cadena de producción. La empresa A se ve menos encorsetada que la B, al realizar una producción de manera más artesanal, contar con empleados más polivalentes y poder cancelar las producciones subcontratadas. Por todo esto, la empresa A mejora a la B en la pregunta 65-68.

La flexibilidad de los salarios no determina diferencias entre las empresas simuladas. Ambas pueden estar dispuestas a subirlos, y condicionadas por las restricciones legales y laborales para bajarlos (preguntas 69-72 y 73-76). Igualmente, las preguntas $77-80$ y 81-84 no producen discrepancias. Las dos empresas, de acuerdo con los sindicatos, pueden extender o reducir el número de horas trabajadas gracias a las horas extras, en un caso, y los expedientes de regulación, vacaciones anticipadas, etc., en otro caso. Respecto al análisis anterior, la empresa A tiene la ventaja del "colchón" de la subcontratación y la parcial red comercial a comisión; sin embargo, cuenta con el inconveniente del elevado coste de la hora/hombre extra en fábrica, que es superior al de la empresa B.

Ante una huelga prevista para dentro de un mes las dos empresas responden bastante bien gracias a la posibilidad de almacenar productos terminados (pregunta 85-88). La empresa A tiene una plantilla formada por operarios de mayor cualificación y con mayor grado de intercambiabilidad que la $B$. Esto determina que la flexibilidad funcional sea superior en la empresa A, como se pone de manifiesto en la pregunta 89-92.

Por último, no se observan diferencias en el grupo de preguntas relacionadas con la flexibilidad geográfica, siendo más bien rígidas ambas empresas (preguntas 93 a 96). Como resumen de la simulación llevada a cabo puede concluirse que la empresa $A$ es más flexible que la empresa $B$, y que esta ventaja se agudiza cuando se tiene en consideración el área comercial y productiva, no siendo el mismo caso cuando se analizan las flexibilidades financiera y laboral.

\section{BIBLIOGRAFÍA}

AAKER, D. A.; MASCARENHAS, B. The Need for Strategic Flexibility. The Journal of Business Strategy, [S.I.], v. 5, n. 2, p. 74-82, 1984.

ABERNATHY, W. J. The productivity dilemma: roadblock to innovation in the automobile industry. Baltimore: Johns Hopkins University Press, 1978.

ADLER, P. S. Managing flexible automation. California Management Review, Berkeley , v. 20, n. 1, p. 34-56, 1988.

ALBIZU, E. Flexibilidad laboral y gestión de los recursos humanos. Barcelona: Ariel, Barcelona, 1997

ANDERSON, J. C.; NARUS, J. A. Capturing the value of supplementary services. Harvard Business Review, Boston, v. 73, n. 1, p. 75-83, Jan. 1995.

ANDREU, R.; RICART, J. E.; VALOR, J. La organización en la era de la información. Barcelona: McGraw-Hill; IESE, 1996.

ANDREWS, K. El concepto de estrategia de la empresa. Pamplona: EUNSA, Pamplona, 1977. 
ANSOFF, H. I. La estrategia de la empresa. Pamplona : EUNSA, Pamplona, 1976.

ANSOFF, H. I.; BRANDENBURG, R. G. A language for organization design. Management Science, Linthicum , v. 17, n. 12, p. 705-731. Ago. 1971.

ANSOFF, H. I.; MCDONNELL, E. J. La dirección estratégica en la práctica empresarial. Wilmington: Delaware Addison-Wesley Iberoamericana, 1997.

AOKI, M. La Estructura de la economía japonesa. Méjico: Fondo de Cultura Económica, 1990.

ATKINSON, J. Manpower strategies for flexible organizations. Personnel Management, London, p. 28-31, Aug. 1984.

ATKINSON, J. Flexibility or fragmentation?: the United Kingdom labour market in the eighties. Labour and Society, [S. I.], v.. 12, n. 1, p. 87-105, 1987.

ATKINSON, J. Two views on a 'farewell to flexibility?'. welcome to what?". Work, Employment \& Society, Durham , v. 6, n. 2, p. 309-310, 1992.

ATKINSON, J.; GREGORY, D. A flexible future: Britain's dual labour force. Marxism Today, [S.I. ], p. 12-17, April 1986.

ATKINSON, J.; MEAGER, N. Is Flexibility just a flash in the pan?. Personnel Management, London, p. 26-29, Sept. 1986.

BAHRAMI, $\mathrm{H}$. The emerging flexible organization: perspectives from Silicon Valley. California Management Review, Berkeley, v. 34, n. 4, p. 33-52, 1992.

BAÑEGIL, T. M. El Sistema just in time y la flexibilidad de la producción. Madrid: Pirámide, 1993.

BARNARD, C. Las funciones de los elementos dirigentes. Madrid: Instituto de Estudios Políticos, 1959

BARTLETT, C. A.; GHOSHAL, S. Changing the role of top management: beyond system to people. Harvard Business Review, Boston, p. 132-142, May/ June 1995.

BECATTINI, G. Dal 'settore' industriale al 'distretto' industriale: alcune considerazioni sull'unità di indagine dell'economia ilndustriale. Rivista di Economia e Politica Industriale, [S.I.], n. 1, ene-abr, p. 7-21, 1979.

BECATTINI, G. Mercato e forze locali: il distretto industriale. Bologna: Il Mulino, 1987. BECATTINI, G. Modelli Locali di Sviluppo. Bologna: II Mulino, 1989.

BECATTINI, G. Dall'Impresa alla quasi-comunità: dubbi e domande. Economia e Politica Industriale, [S.I.], n. 68, p. 7-12, 1990.

BEER, S. Fanfare for effective freedom: cybernetic praxis in government. En: BEER, S. (Ed.). Platform for change: a message from Stafford Beer. London: Wiley, 1975.

BELLANDI, M . Capacità innovativa diffusa e sistemi locali di imprese. En: BECATTINI, G. (ed.). Modelli Locali di Sviluppo. Bologna: II Mulino, 1989.

BENKO, G.; LIPIETZ, A. ( Ed.) Las regiones que Ganan. Valencia: Edicions Alfons El Magnànim, 1994. 
BENKO, G.; LIPIETZ, A. El nuevo debate regional. En: BENKO, G.; LIPIETZ, A. ( Ed.) Las regiones que Ganan. Valencia: Edicions Alfons El Magnànim, 1994.

BLOIS, K. J. Matching new manufacturing technologies to industrial markets and strategies. Industrial Marketing Management, [S.I.], v. 14, n. 1, p. 43-47, Feb. 1985.

BLYTON, P. Flexible times? recent developments in temporal flexibility. Industrial Relations Journal, v. 23, n. 1, p. 26-36, 1992.

BOYER, R. Théorie de la eégulation, une analyse critique, la découverte. París: [s.n], 1992.

BOYER, R. Las alternativas al fordismo. de los años 80 al siglo XXI. En: BENKO, G.; LIPIETZ, A. ( Ed.) Las regiones que Ganan. Valencia: Edicions Alfons El Magnànim, 1994.

BROWNE, J. et al. Classification of flexible manufacturing systems. The FMS Magazine, [S.I.], v. 2, n. 2, p. 114-117, 1984.

BURNS, T.; STALKER, G.M. The management of innovation. London: Tavistock, 1961. BURROWS, R.; GILBERT, N.; POLLERT, A. Introduction: fordism, postfordism and economic flexibility. En: Gilbert, N., Burrows, R.; Pollert, A. (Ed.): Fordism and flexibility: divisions and change. Basingstoke: MacMillan, 1992.

CARLSSON, B. Flexibility and the theory of the firm. International Journal of Industrial Organization, [S.I], v. 7, p. 179-203, 1989.

CASEY, B. Survey evidence on trends in 'non-standard' employment. En: Pollert, A. (Ed.): Farewell to flexibility? Oxford : Blackwell, 1991.

CLUTTERBUCK, D.; CRAINER, S. Los maestros del management. Barcelona: Grijalbo, 1991.

CONNELL, R.; PHILLIPS, B. Finding funds for small firms. Management Today, [S.I.], p. 143-164, nov .1988.

CORIAT, B. El taller y el cronómetro. Madrid: Siglo XXI, 1982.

COURLET, C.; PECQUEUR, B. Sistemas industriales locales en Francia: un nuevo modelo de desarrollo. En: BENKO, G.; LIPIETZ, A. ( Ed.) Las regiones que Ganan. Valencia: Edicions Alfons El Magnànim, 1994

COX, T. Towards the measurement of plant flexibility. Production and Inventory Management, v. 30, n. 1, p. 68-72, 1989.

CROSS, M. Changes in working practices in U.K.: manufacturing 1981-88", Industrial Relations Review and Report, v. 415, p. 2-10, 1988.

CUERVO, A. La dirección estratégica de la empresa. En: CUERVO, A. (Ed.). Dirección de empresas de los noventa. Madrid: Civitas, 1995.

CUSUMANO, M. A. The limits of 'lean'. Sloan Management Review, Cambridge, v. 35, n. 4, p. 27-32, Summer 1994.

CHANDLER, A. D. Strategy and structure. Cambridge: MIT Press, Cambridge, 1962. CHIAVENATO, I. Introducción a la teoría general de la administración. Bogotá: McGraw-Hill, 1981. 
DAFT, R. L.; LEWIN, A. Y. Where are the theories for the "new" organizational forms? an editorial essay. Organization Science, v. . 4, n. 4, p. i-vi, 1993.

DONALDSON, G. Strategy for financial mobility. Boston : Harvard University, 1969.

DORE, R. Flexible rigidities: industrial policy and structural adjustment in the japanese economy, 1970-80. Londres: The Athlone Press, 1986.

DRUCKER, P. F. La teoría de la fabricación que surge ahora. Harvard-Deusto Business Review, 1st. trim., p. 13-22, 1991.

DRUCKER, P. F. La sociedad poscapitalista. Barcelona: Apóstrofe, 1993.

DUNNING, J.H. Reappraising the eclectic paradigm in a age of alliance capitalism. Journal of International Business Studies, v. 26, n . 3, p. 461-491, 1995.

ECCLES, H. E. Logistic and the national defence. London: Stackpole, 1959.

ELGER, T. Task flexibility and the intensification of labour in U.K. manufacturing in the 1980's. En: Pollert, A. (Ed.). Farewell to Flexibility? Oxford: Blackwell, 1991.

EPPINK, D. J. Planning for strategic flexibility. Long Range Planning, v. 11, n. 4, p. 915, 1978.

EVANS, J. S. Strategic flexibility for high technology manoeuvres: a conceptual framework. Journal of Management Studies, v. 28, n. 1, p. 69-89, 1991.

FERRÉ, R. La fábrica flexible. Barcelona: Marcombo, 1988.

FIEGENBAUM, A.; KARNANI, A. Output flexibility: a competitive advantage for small firms. Strategic Management Journal, v. 12, n. 2, p. 101-114, 1991.

FINA, L. El problema del paro y la flexibilidad del empleo: informes sobre un debate. Madrid. Ministerio de Trabajo y Seguridad Social, Centro de Publicaciones, 1991.

GALBRAITH, J. A. The value-adding corporation: matching structure with strategy. En: GALBRAITH, J. A., LAWLER III, E. E., Y Asociados (Ed.): Organizing for the future: the new logic for managing complex organization. San Francisco: Jossey-Bass Publishers, 1993a.

GALBRAITH, J. A. The business unit of the future. En: GALBRAITH, J. A., LAWLER III, E. E., y Asociados (eds.): Organizing for the future: the new logic for managing complex organization. San Francisco: Jossey-Bass Publishers, 1993b.

GALBRAITH, J. A.; LAWLER III, E. E., y Asociados (eds.): Organizing for the future: the new logic for managing complex organization. San Francisco: Jossey-Bass Publishers, 1993.

GALBRAITH, J. A.; LAWLER III, E. E. Challenges to the established order. En: GALBRAITH, J. A., LAWLER III, E. E., y Asociados (eds.): Organizing for the future: the new logic for managing complex organization. San Francisco: Jossey-Bass Publishers, 1993.

GALBRAITH, J. R.; SCHOEMAKER, P. J. Tecnología y proyectos de organización: valoración directiva. En: HAX, A. C. (Ed.). Dirección de operaciones en la empresa. Barcelona: Hispano Europea, 1983. 
GALLEGO, J. R. Empresa e historia: entorno institucional y macroeconomía. En: LLOPIS, J. B. et al . Elementos básicos de economía. Valencia: Tirant lo Blanch, 2001.

GARCÍA-GARCÍA, C. E. Una teoría evolucionista de la empresa: la esperanza genética. Revista Europea de Dirección y Economía de la Empresa, v. 5, n. 3, p. 57-72, 1996.

GEARY, J. F. Employment flexibility and human resource management: the case of three american electronics plants. Work, Employment \& Society, Durham, v. 6, n. 2, p. 251270, 1992.

GERWIN, D. Do's and dont's of computerized manufacturing. Harvard Business Review, Boston, v. 60, n. 2, p. 107-116, 1982.

GERWIN, D. An Agenda for research on the flexibility of manufacturing processes. International Journal of Operations and Production Management, v.. 7, n. 1, p. 3849, 1987.

GERWIN, D. Manufacturing flexibility in the CAM era. Business Horizons, v. 32, n. 1, p. 78-84, 1989.

GERWIN, D. Manufacturing flexibility: a strategic perspective. Management Science, Linthicum , v. 39, n. 4, p. 395-410, 1993.

GERWIN, D.; TARONDEAU, J. La flexibilité dans les processus de production: le cas de l'automobile. Revue Française de Gestion, n. 46, juin/août, p. 37-46, 1984.

GHOSHAL, S.; BARTLETT, C. A. changing the role of top management: beyond structure to processes. Harvard Business Review, Boston, p. 85-96, Jan./ Feb., 1995

GIGCH, J. P. van. Teoría general de sistemas. 2. ed. Méjico: Trillas, 1990.

GILLY, J. P. Dinamiche industriali e meso-analisi: il caso dei sistemi locali di innovazione. L'Industria, ano 15, n. 2, apr./ giug. , p. 295-309, 1994.

GOLDHAR, J.; JELINEK, M. Computer integrated flexible manufacturing: organizational, economic and strategic implications. Interfaces, Linthicum, v. 15, n. 3, p. 94-105, May/ June 1985.

GOLDMAN, S. M. Portfolio choice and flexibility. Journal of Monetary Economics, v. 4, p. 263-279, 1978.

GRANT, R. M. Dirección estratégica. Madrid: Cívitas, 1996.

HAKIM, C. 'Core and periphery in employers' workplace strategies: evidence from the 1987 ELUS Survey. Work, Employment \& Society, Durham, v. 4, n. 2, p. 157-188, 1990.

HAMEL, G.; PRAHALAD, C. K. Propósito Estratégico. En: THOMPSON, A. A. JR.; STRICKLAND, A. J. III, Dirección y Administración Estratégicas. Wilmington, Delaware: Addison-Wesley Iberoamericana, 1994.

HAMEL, G.; PRAHALAD, C. K. Compitiendo por el futuro: estrategia crucial para crear los mercados del Mañana. Barcelona: Ariel, 1995.

HANSSEN-BAUER, J.; SNOW, C. Responding to hypercompetition: the structure and processes of a regional learning network organization. Organization Science, v. 7, n. 4, p. 413-427, 1996. 
HARRIGAN, K. R. Strategic flexibility: a management guide for changing times. Lexington: Lexington Books, 1985.

HARRISON, B.; KELLEY, M. R. Outsourcing and the search for flexibility. Work, Employment \& Society, Durham, v. 7, n. 2, p. 213-235, 1993.

HART, A. G. Anticipations, business planning and the cycle. Quarterly Journal of Economics, p. 272-293, Feb. 1937

HAYES, R. H.; PISANO, G. P. Beyond world class manufacturing: the new manufacturing strategy. Harvard Business Review, Boston, v. 71, n. 2, p. 77-87, 1994.

HAYES, R. H.; WHEELWRIGHT, S. C. The dynamics of process-product life cycles. Harvard Business Review, Boston, v. 57, n. 2, p. 15-22, 1979.

HEDLUND, G. A model of knowledge management and the n-form corporation. Strategic Management Journal, v. 15, p. 73-90, 1994.

HIRST, E. Benefits and cost of flexibility: short-lead-time power plants. Long Range Planning, v. 23, n. 5, p. 106-115, 1990.

HIRST, P.; ZEITLIN, J. (Ed.): Reversing industrial decline?: industrial structure and policy in britain and her competitors. Oxford: Berg, 1989.

HIRST, P.; ZEITLIN, J. Especialización flexible vs. postfordismo: teoría, evidencia e implicaciones políticas. Papers de Seminari, n . 33/34, p. 1-81, 1991

HUNTER, L.; et al . The flexible firm: strategy and segmentation. British Journal of Industrial Relations, v. 31, n. 3, p. 383-407, 1993.

HYMAN, R. Plus ça change? the theory of production and the production of theory. En: Pollert, A. (Ed.). Farewell to flexibility?. Oxford: Blackwell, 1991.

HYMAN, R.; STREECK, W. (Ed.): Nuevas tecnologías y relaciones industriales. Madrid: Ministerio de Trabajo y Seguridad Social, 1993.

IMAI, K., NONAKA, I., TAKEUCHI, H. Managing the new product development process: how japanese companies learn and unlearn. En: CLARK, K. B.; HAYES, R. H.; LORENZ, C. (Ed.). The uneasy alliance: managing the productivity-technology dilemma. Boston: Harvard Business School Press, 1985.

JAIKUMAR, R. Flexible manufacturing systems: a managerial perspective. Boston: Harvard Business School, 1984.

JAIKUMAR, R. Postindustrial manufacturing. Harvard Business Review, Boston, v..64, n. 6, p. 69-76, Nov./Dec, 1986.

JONES, R. A.; OSTROY, J. M. Flexibility and uncertainty. Review of Economic Studies, n. 164, p. 13-32, 1984.

KAGONO, T., et al. (1985): Strategic vs. evolutionary management: a U.S.-Japan comparison of strategy and organization. Amsterdam: North Holland, 1985.

KANTER, R. M. The change masters. New York: Simon \& Schuster, 1983.

KAST, F. E.; ROSENZWEIG, J. E. Administración en las organizaciones: enfoque de sistemas y de contingencias. México: McGraw-Hill, 1992. 
KOENES, A. Las tres armas estratégicas de la pequeña empresa. Madrid: Díaz de Santos, 1994.

KOTHA, S. Mass customization: implementing the emerging paradigm for competitive advantage. Strategic Management Journal, v. 16, p. 21-42, 1995

KOTHA, S.; ORNE, D. Generic manufacturing strategies: a conceptual synthesis. Strategic Management Journal, v. 10, n.. 3, p. 211-231, 1989.

KOTLER, P. From mass marketing to mass customization. Planning Review, v. 17, p. 10-13, 1989.

KRAFCIK, J. F. Triumph of the lean production system. Sloan Management Review, Cambridge , v. 30, n. 1, p. 41-52, 1988.

KRIJNEN, H. G. The flexible firm. Long Range Planning, v. 12, p. 63-75, Apr. 1979.

KULATILAKA, N. The value of flexibility: the case of a dual-fuel industrial steam boiler. Financial Management, v. 22, n. 3, p. 271-280, 1993.

KUSIAK, A. Flexible manufacturing systems: a structural approach. International Journal of Production Research, v. 23, n. 6, p. 1057-1073, 1985.

LASH, S.; URRY, J. The end of organized capitalism. Cambridge: Polity, 1987.

LASZLO, E. A general systems view of evolution and invariance. General Systems, v. 19, p. 37-43, 1974.

LASZLO, E.; LASZLO, C. Navigare nella turbulenta. Milano: FrancoAngeli, 1997.

LAWRENCE, P. R.; LORSCH, J. W. Organization and Environment. Boston: Harvard Business School, Division of Research, 1976.

LEHNER, F. Anthropocentric production systems: the european response to advanced manufacturing and globalization. Brussels : CEE FAST, 1991. (APS research papers series, v. 4).

LEONARD-BARTON, D. Core capabilities and core rigidities: a paradox in managing new product development. Strategic Management Journal, v. 13, p. 111-125, 1992.

LEVITT, T. The globalization of markets. Harvard Business Review, Boston, v. 61, n. 3, p. 92-102, Mayo/June 1983.

LILIENFELD, R. Teoría de sistemas: origenes y aplicaciones en ciencias sociales. México: Trillas, 1991.

LORENZONI, G.; BADEN-FULLER, CH. Creating a strategic center to manage a web of partners. California Management Review, v. 37, n. 3, p. 146-163, 1995.

LUND, R.; GJERDING, A.N. The flexible company: innovation, work organization and human resource management. DRUID Working Paper, n. 96-17, Dec. 1996.

LUTHANS, F. Introducción a la administración: un enfoque de contingencias. México: McGraw-Hill, 1980.

MARGINSON, P. Change and continuity in the employment structure of large companies. En: POLLERT, A. (Ed.). Farewell to Flexibility?, Oxford: Blackwell, 1991. 
MARSHALL, A. Industry and trade. London: MacMillan, 1891.

MARTÍN, A. Flexibilidad laboral y planificación de personal. Anales de Economía y Administración de Empresas, n. 1, p. 89-108, 1993.

MASCARENHAS, B. Planning for flexibility. Long Range Planning, v. 14, n. 5, p. 7882, 1981.

MASON, S. P. Valuing financial flexibility. En: FRIEDMAN, B. M. (Ed.). Financing corporate capital formation. Chicago: University of Chicago Press, 1986.

MENGUZZATO, M.; RENAU, J. J. La dirección estratégica de la empresa: un enfoque innovador del management. Barcelona: Ariel, 1991.

MILES, R. E. Adapting to technology and competition: a new industrial relations system for the 21st century, California Management Review, v. 31, n. 2, p. 9-28, 1989.

MILES, R. E.; SNOW, C. C. Fit, failure \& the hall of fame. California Management Review, v. 26, n. 3, p. 10-28, 1984.

MINTZBERG, H. La estructuración de las organizaciones. Barcelona: Ariel, Barcelona, 1991.

MINTZBERG, H.; AHLSTRAND, B.; LAMPEL, J. Strategy safari. New York: Prentice Hall, 1998.

MINTZBERG, H.; QUINN, J.B. (Ed.). El proceso estratégico. México: Prentice Hall, 1993.

MORENO-LUZÓN, M. D.; PERIS, F. J.; SANTONJA, F. J. Quality management in small and medium-sized companies and strategic management. En: MADU, C. N. (Ed.). Handbook of total quality management, Boston: Kluwer Academic Pub., 1998.

MORENO-LUZÓN, M. D.; PERIS, F. J. Strategic approaches, organizational design and quality management: integration in a fit and contingency model. International Journal of Quality Science, v. 3, n. 4, p. 328-347, 1998.

NATIONAL ECONOMIC DEVELOPMENT OFFICE. Changing working patterns: how companies achieve flexibility to meet new needs. Londres, 1986.

NELSON, R. Production sets, technological knowledge, and R\&D: fragile and overworked constructs for analysis of productivity growth?. American Economic Review, v. 70, n. 2, p. 62-67, 1980.

NONAKA, I. Managing the firms as information creation process. En: Meindl, J. (Ed.). Advances in information processing in organizations. Greenwich, Conn: JAI Press, 1991. v. 4.

NONAKA, I. Toward middle-up-down management: accelerating information creation. Sloan Management Review, Cambridge, p. 9-18, 1988a.

NONAKA, I. Creating organizational order out of chaos: self-renewal in japanese firms. California Management Review, p. 57-73, 1088b.

NONAKA, I. The knowledge-creating company. Harvard Business Review, Boston, p. 96-104, Nov./Dec. 1991. 
NONAKA, I. A dynamic theory of organizational knowledge creation. Organization Science, v. 5, n. 1, p. 14-37, 1994.

NONAKA, I.; TAKEUCHI, H. The knowledge-creating company: how japanese companies create the dynamics of innovation. New York: Oxford University Press, 1995.

O'REILLY, J. Where do you draw the line? functional flexibility, training \& skill in britain \& france. Work, Employment \& Society, Durham, v. 6, n. 3, p. 369-396, 1992.

OCDE. Políticas de mercado de trabajo en los noventa. Madrid, Centro de Publicaciones del Ministerio de Trabajo y Seguridad Social, 1991.

OHMAE, K. La lógica global de las alianzas estratégicas. En: MINTZBERG, H.; QUINN, J.B. (Ed.). El Proceso Estratégico. México: Prentice Hall, 1993.

OHMAE, K. El despliegue de las economías regionales: oportunidades y desafío empresarial. Bilbao: Deusto, 1996.

$\mathrm{OUCHI}$, W. Markets, bureaucracies, and clans. Administrative Science, v. 25, p. 129141, quarterly 1980.

OUCHI, W. La teoría Z. 4. ed. Barcelona: Orbis, 1985.

PENN, R. Flexibility in Britain during the 1980's: recent empirical evidence. En: GILBERT, N.; BURROWS, R.; POLLERT, A. (Ed.): Fordism and flexibility: divisions and change. Basingstoke: MacMillan, 1992.

PERIS, F. J. El 'soporte' de la estrategia. los enfoques de costes de transacción y de configuraciones-contingencias en el diseño de organizaciones. En: Cuervo, A. (ed.). Dirección de Empresas de los Noventa. Madrid, Civitas, 1995.

PERIS, F. J. Notas sobre estrategia y estructura. Valencia: Universidad de Valencia, 1990. Documento de trabajo no publicado

PERIS, F. J.; Fernández, R.; Tarazona, F. Curso de dirección y organización de empresas. [S.I.]: Tirant lo Blanch, 1995.

PERIS, F. J.; HERRERA, J. Diseño de organizaciones en las nuevas formas organizativas y sus implicaciones en los recursos humanos de la empresa. Revista Europea de Dirección y Economía de la Empresa, v. 7, n. 3, p. 99-120, 1998.

PETERS, T. J.; WATERMAN JR., R. H. En busca de la excelencia. 5. ed. Barcelona: Folio, 1991.

PINE, B. J.; VICTOR, B.; BOYNTON, A. C. Making mass customization work. Harvard Business Review, Boston, v. 71, p. 108-119, 1993.

PIORE, M. J.; SABEL, C. F. The second industrial divide: basic books. New York: [S.n.], 1990.

POLANYI, M. The tacit dimension. Londres: Routledge \& Kegan Paul, 1966. Citado en Nonaka (1994).

POLLERT, A. The 'flexible firm': fixation or fact?. Work, Employment \& Society, Durham, V. 2, n. 3, p. 281-316, 1988.

POLLERT, A. (Ed.). Farewell to flexibility? Oxford: Blackwell, 1991. 
POLLERT, A. The orthodoxy of flexibility. En: POLLERT, A. (Ed.). Farewell to flexibility? Oxford: Blackwell, 1991.

PORTER, M. E. Competitive Advantage. New York: Free Press, 1987.

PRAHALAD, C. K.; HAMEL, G. The core competence of the corporation. Harvard Business Review, Boston, v. 68, n. 3, p. 79-91, 1990.

PROCTER, S. J. et al. Flexibility, politics \& strategy: in defence of the model of the flexible firm. Work, Employment \& Society, Durham, v. 8, n. 2, p. 221-242, 1994.

SAFÓN, V. (1996). Las redes de empresas: una fuente de ventajas competitivas para las pymes de la vall d'albaida. In: CONGRÉS D'ESTUDIS DE LA VALL D'ALBAIDA, 1. , 1996, Valencia. Actes... Valencia: Aielo de Malferit 1996.

SAFÓN, V. ¿Del fordismo al postfordismo? el advenimiento de los nuevos modelos de organización industrial. In: CONGRESO DE CIENCIA REGIONAL DE ANDALUCÍA, 1., 1997, Jerez de la Frontera. Actas... Jerez de la Frontera: [s.n.], 1997a.

SAFÓN, V. Estudio de la cooperación interempresarial en los distritos industriales ortodoxos. In: CONGRESO DE CIENCIA REGIONAL DE ANDALUCÍA, 1. 1997. Jerez de la Frontera. Actas... Jerez de la Frontera: [s.n.], 1997b.

SAFÓN, V. La Cooperación entre empresas de pequeña dimensión: el modelo de los distritos industriales. Revista Asturiana de Economía, n. 9, p. 174-201, 1997c.

SAFÓN, V. El crecimiento vía red: importancia del territorio de pertenencia en las redes de empresas. In: CONGRESO NACIONAL DE LA ASOCIACIÓN CIENTÍFICA DE ECONOMÍA Y DIRECCIÓN DE LA EMPRESA, 7., 1997, Almería. Actas... Almería: ACEDE, 1997d. V. 2, p. 419-428.

SAFÓN, V. Creación y desarrollo del conocimiento en la organización. Revista Europea de Dirección y Economía de la Empresa, v. 6, n. 2, p. 115-126, 1997e

SAFÓN, V. Las redes de pymes en los distritos industriales españoles: propuestas de desarrollo y consolidación. Alta Dirección, n. 201, p. 19-25, 1998.

SAFÓN, V. La flexibilidad de la empresa: teoría, cuantificación y práctica. Valencia: Promolibro, 2000.

SÁNCHEZ, R. Strategic flexibility in product competition. Strategic Management Journal, v. 16, p. 135-159, 1995.

SANCHIS, J. R.; SAFÓN, V. Las redes de empresas: teoría y aplicación a las cooperativas de trabajo asociado valencianas. Economía Industrial, n. 318, p. 173-184, 1997.

SARABIA, J. Ma . Un boceto de empresa 2000. En: Cuervo, A. (Ed.). Dirección de Empresas de los Noventa. Madrid Civitas, 1995.

SARFATI, H.; KOBRIN, C. (Ed.): La flexibilidad del mercado de trabajo: antología comparada. Madrid: Ministerio de Trabajo y Seguridad Social, Centro de Publicaciones, 1992.

SAXENIAN, A. (1995): "Redes Regionales y Adaptación Industrial en Silicon Valley y la Ruta 128", Revista Asturiana de Economía, nº. 4, pp. 231-253. 
SCHONBERGER, R. J. Frugal Manufacturing. Harvard Business Review, Boston, v. 65, n. 5, p. 95-100, 1997.

SEMLER, R. Radical: el éxito de una empresa sorprendente. Barcelona:Ediciones Gestión 2000.

SHAPIRO, B. P. Can marketing and manufacturing coexist?.Harvard Business Review, Boston, p. 416-427, Sept./Oct. 1977.

SILVERBERG, G.; DOSI, G.: ORSENIGO, L. Innovation, diversity and diffusion: a selforganizing model. The Economic Journal, v. 98, n. 393, p. 1032-1054, 1988.

SKYRME, D. J. Flexible working building a lean and responsive organization. Long Range Planning, v. 27, n. 5, p. 98-110, 1994.

SLACK, N. Flexibility as a manufacturing objective. International Journal of Operation \& Production Management, v. 3, n. 3, p. 4-13, 1983.

SLACK, N. Flexibility of manufacturing systems. International Journal of Operation \& Production Management, v. 7, n. 4, p. 35-45, 1987.

STALK, G.; EVANS, P.; SHULMAN, L. Competing on capabilities: the new rules of corporate strategy. Harvard Business Review, Boston, v. 70, n. 2, p. 57-69, 1992.

STIGLER, G. Production and distribution in the short run. The Journal of Political Economy, v. 47, n. 3, p. 305-327, June 1939.

STRATEGOR . Estrategia, estructura, decisión, identidad: política general de la empresa. Barcelona: Biblio Empresa, 1995.

SUÁREZ, F. F.; CUSUMANO, M. A.; FINE, Ch. H. An empirical study of flexibility in manufacturing. Sloan Management Review, Cambridge, v. 37, n. 1, p. 25-32, 1995.

SULLIVAN, J. J.; NONAKA, I. The application of organizational learning theory to japanese and american management. Journal of International Business Studies, v. 17, n. 3, p. 127-147, 1986.

SWAMIDASS, P. M. Manufacturing strategy: a selected bibliography. Journal of Operations Management, v. 8, n. 3, p. 263-277, 1989.

TAKEUCHI, H.; NONAKA, I. The New new product development game. Harvard Business Review, Boston, p. 137-146, Jan./Feb.1986.

TEECE, D.; PISANO, G; SHUEN, A. Dynamic capabilities and strategic management. Strategic Management Journal, v. 18, n. 7, p. 509-533, 1997.

THOMPSON, J. D. Organizations in action. New York: McGraw-Hill, 1994.

UPTON, D. M. The management of manufacturing flexibility. California Management Review, v. 36, n. 2, p. 72-89, 1994.

VAN ALSTYNE, M. The state of network organization. Journal of Organizational Computing, v. 7, n. 3, 1997.

VENCE, X. Economía de la innovación y del cambio tecnológico. Madrid: Siglo Veintiuno, 1995. 
VENTURA, J. Análisis dinámico de la estrategia empresarial: un ensayo interdisciplinar. Oviedo: Servicio de Publicaciones de la Universidad de Oviedo, 1996.

VOLBERDA, H. W. Building the flexible firm. New York: Oxford University Press, 1998.

VOLTES, P. La teoría general de sistemas. Barcelona: Hispano Europea, 1978.

VON KROGH, G.; ROOS, J.; SLOCUM, K. An essay on corporate epistemology. Strategic Management Journal, v. 15, p. 53-71, 1994.

VON KROGH, G.; VICARI, S. An autopoiesis approach to experimental strategic learning. En: LORANGE, B., et al. (Ed.) Implementing strategic processes: change, learning and co-operation. Londres: Blackwell, 1993.

WATERMAN, R. H. Adhocracy: the power to change. New York: Norton, 1991.

WHEELWRIGHT, S. C.; HAYES, R. H. Competing through manufacturing. Harvard Business Review, Boston, p. 99-145, Jan./Feb. 1985.

WHIPP, R.; ROSENFELD, R.; PETTIGREW, A. Managing strategic change in a mature business. Long Range Planning, v. 22, n. 6, p. 92-99, 1999.

WOODWARD, J. Industrial organization: theory and practice. Oxford: Oxford University Press, 1965.

ZELENOVIC, D. M. Flexibility: a condition for effective production systems. International Journal of Production Research, v. 20, n. 3, p. 319-337, 1982.

\title{
FLEXIBILITY IN THE SMALL SIZE COMPANY: A STUDY ON THE DEVELOPMENT OF AN OPERATIONAL AND QUANTITATIVE CONCEPT
}

\begin{abstract}
In this article we present an approach of a theme that has been largly argued among the academical world: the flexibility of companies regarding the context variable. More specifically, the paper presented a model to quantify the flexibility strategy of a small size company. Considering the features of commercial, productive and financial resources, an operational and quantitative model of firm flexibility have been developed.
\end{abstract}

Key-words: small size company, flexibility, context variables. 